Distribution Category:

LMFBR Safety (UC-79p)

ANL $-77-2$

ARGONNE NATIONAL LABORATORY

9700 South Cass Avenue

Argonne, Illinois 60439

\title{
A GENERALIZED PARAMETRIC MODEL FOR TRANSIENT GAS RELEASE AND \\ SWELLING IN OXIDE FUELS
}

by

E. E. Gruber

Reactor Analysis and Safety Division

This teport wat pirputice -

wonsored by the prrpurde as an sccount of work

the United Stater not the Government. Neithet

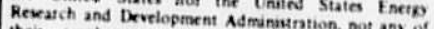

their employess. not any of their contractors.

wartanty, captes ot implempioyces, makes atiy

batility of reiponsbitity for the of asumes any lexal

ot usfulnce of aty information. appartis preients

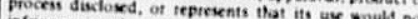

infrner privately ounned rights.

January 1977 

TABLE OF CONTENTS

Page

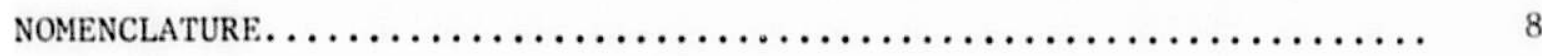

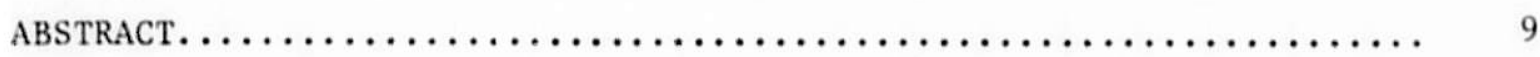

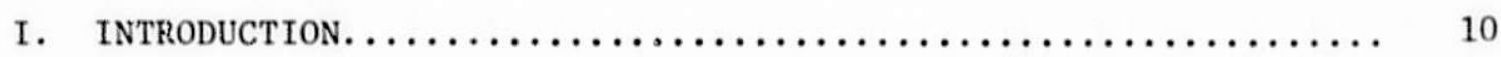

II. MECHANISTIC ANALYSIS: THE FRAS CODE.................. 12

A. Van der Waals Equation of State.................. 14

B. Bubble Migration by Surface Diffusion............... 14

C. Effect of Swelling on Coalescence Probability.......... 17

D. Product-bubble Classification.................... 19

E. FRAS Calculation of the Evolution of the Bubble-size

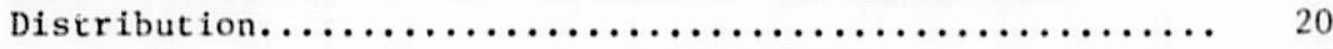

F. Gas Release from Grains to Grain Boundaries............ 21

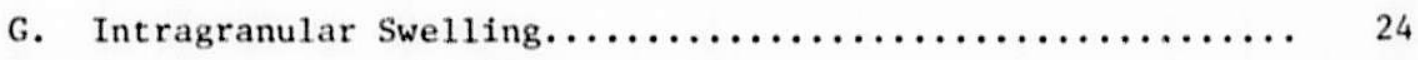

H. Selection of Material Parameters.................. 25

III. PARAMETRIC SYNTHESIS OF FRAS RESULTS: PFRAS $\ldots \ldots \ldots \ldots \ldots \ldots \ldots .26$

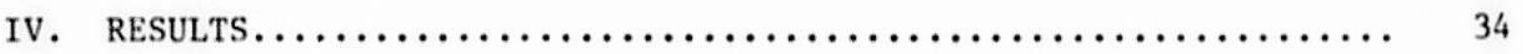

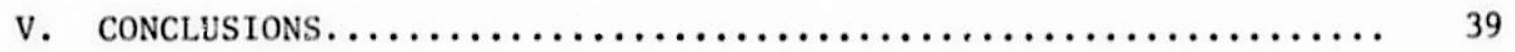

APPENDIX: Calculation of Mobility and Swelling Parameters $\mu$ and $\phi$

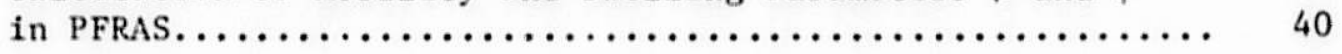

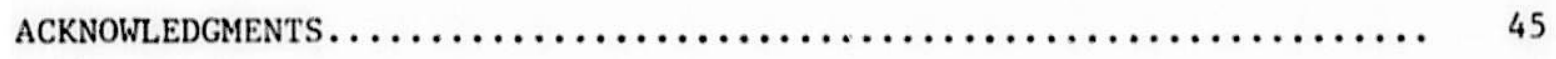

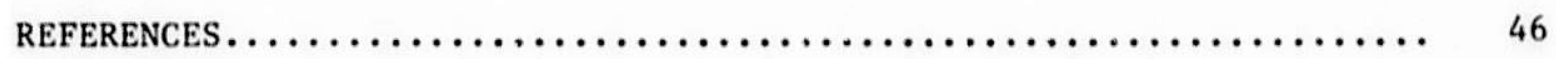




\section{LIST OF FIGURES}

№.

$\underline{\text { Title }}$

Page

1. Schematic Spherical Pore of Radius $r$, Showing Surface-diffusion Mean Jump Distance $\lambda_{s}$ and Effective Jump Distance $\lambda_{s}^{\prime} \ldots \ldots \ldots \ldots$ is

2. Bubble-size Distribution Functions for Idealized Conditions (from Ref. 5) ................................... 20

3. Evolution of Bubble-size Distribution Function for Oxide Fue1

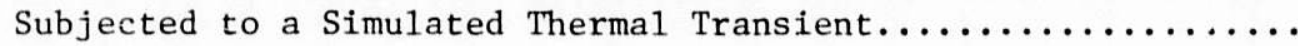

4. Schematic Illustration of Biased-migration Release from a

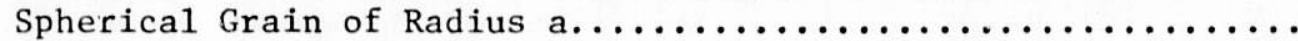

5. Variation in the Standard Distribution Function $Z_{2}$ with Dimensionless Time $\tau^{\prime}$, Showing the Tendency Toward an

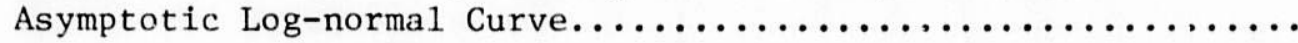

6. Variation of $\mu$ with $\tau$ at $P=0$ for Various Conditions, as Ca1culated with the FRAS Code........................ 30

7. Effect of Pressure on the Variation of $\mu$ with $\tau \ldots \ldots \ldots \ldots \ldots \ldots . . . .30$

8. Variation of $\phi$ with $\tau$ at $P=0$ for Various Conditions........ 32

9. Effect of Pressure on the Variation of $\phi$ with $\tau \ldots \ldots \ldots \ldots \ldots \ldots, 32$

10. The Effect of an Abrupt Change in Pressure, from 0.25 to

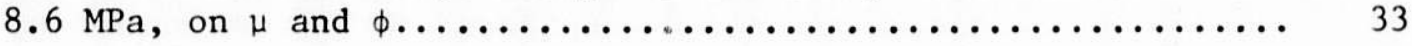

11. Effect of Grain Size on Gas Release and Swelling........... 35

12. Effect of Initial Fission-gas Concentration on Gas Release and

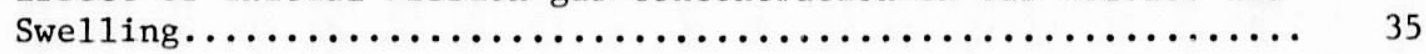

13. Effect of Transient Heating Rate on Gas Release and Swe11ing... 36

14. Effect of Transient Thermal Gradient on Gas Release and

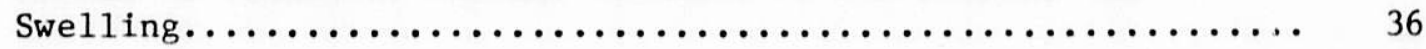

15. Effect of Pressure on Gas Release and Swelling............. 37

16. Effect of Hypothetical Variation in Pressure on Gas Release and Swelling in Fuel Subjected to a Thermal Transient..........

A1. Schematic Illustration of a Grid Element for Interpolation for

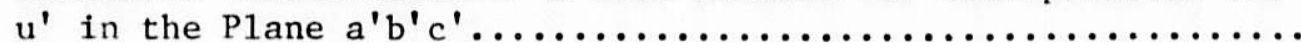


LIST OF TABLES

№.

Title

Paga

A1 $\mu \times 10^{-5}$ for Various $\tau$ and $P \ldots \ldots \ldots \ldots \ldots \ldots \ldots \ldots \ldots \ldots \ldots \ldots \ldots \ldots \ldots \ldots \ldots \ldots$

A2 $\phi \times 10^{30}$ for Various $\tau$ and $P \ldots \ldots \ldots \ldots \ldots \ldots \ldots \ldots \ldots \ldots \ldots \ldots \ldots \ldots \ldots \ldots \ldots \ldots \ldots \ldots$ 
NOMENCLATURE

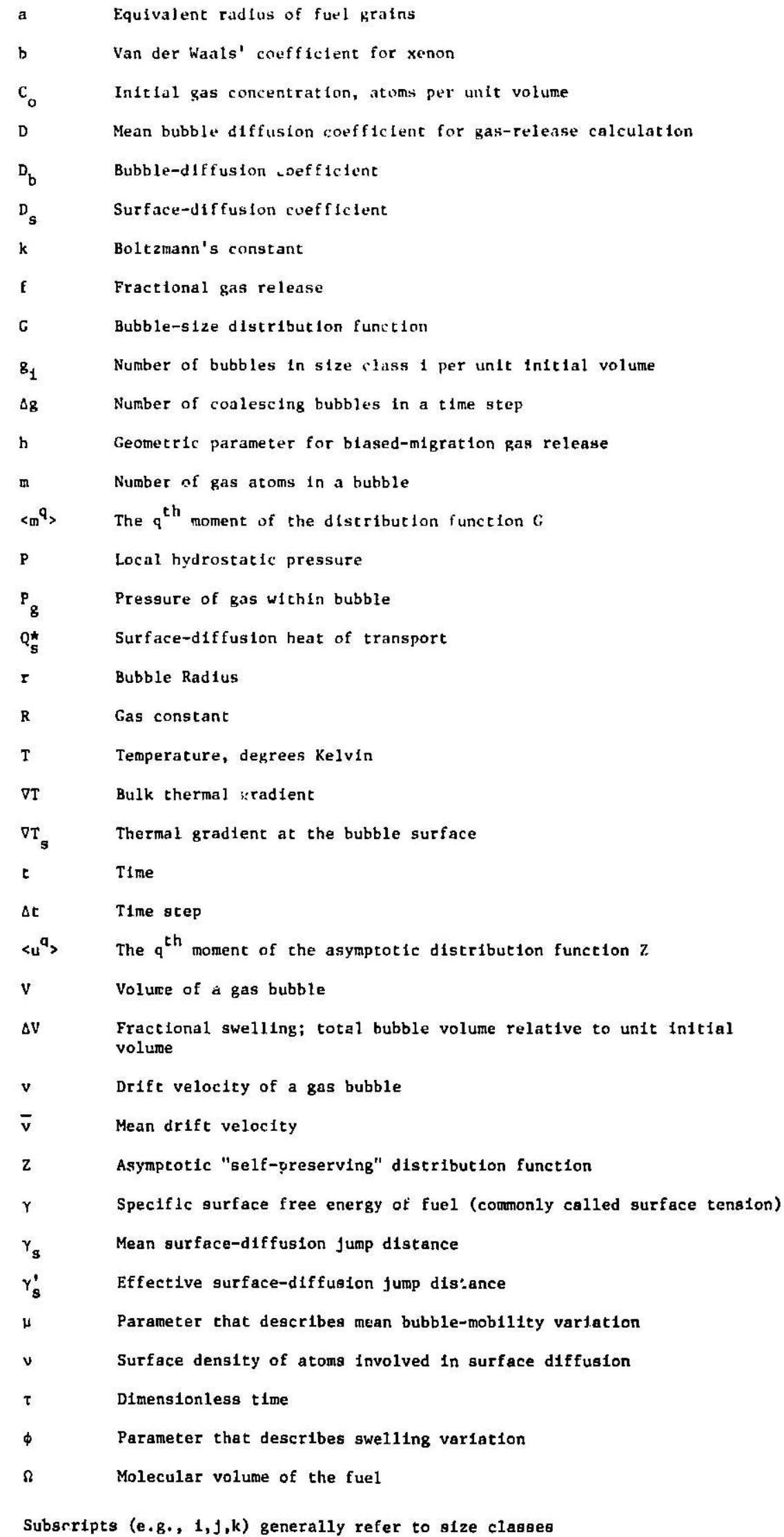


A GENERALIZED PARAMETRIC MODEL FOR TRANSIENT

GAS RELEASE AND SWELLING IN OXIDE FUELS

by

E. E. Gruber

\begin{abstract}
The need for a capability to predict transient fissiongas behavior arises because of the complexity of fission-gas response to transient conditions, the importance of fission gas to fuel. mechanical response, and the prevalent limitations on experimental information relevant to the problem. This report describes a detailed mechanistic analysis of intragranular swelling and release of gas from grains to grain boundaries, both as they result from transient heating.

A generalized parametric model to approximate the results that would be obtained from the more detailed calculations is also described. The need for this model arises from the necessity to consider the fission-gas effects in more general multinode accident-analysis and pin-mechanics codes. For such calculations, the more detailed Fission-gas Release And Swelling (FRAS) code is prohibitive in its demands on computer storage and execution time, while the Parametric FRAS (PFRAS) code reduces these demands by an order of magnitude.

Examples of results of transient calculations are shown for the two codes, both to illustrate the sensitivity of the results to the parameters, and to indicate the level of confidence that can reasonably be ascribed to PFRAS results. The parameters considered include initial gas concentration, grain size, heating rate, thermal gradient, and pressure. The PFRAS model gives a satisfactory approximation to FRAS results for the broad range of parameters surveyed.
\end{abstract}




\section{INTRODUCTION}

Fission gas is an important factor in the response of irradiated oxide fuel to a thermal transient. Gas released to grain boundaries will contribute to fuel breakup or motion and swelling, and the gas retained within the grains can cause swelling of the fuel. These fission-gas effects interact with fuel stresses, and must be considered in realistic pin mechanics models.

Transient fission-gas behavior cannot be adequately characterized from experiments alone. Experiments are not only difficult to perform, and costly, but even more difficult to analyze completely. Some measurements of gas behavior can be made during the transient. For example, the gas release from the pin can be deduced as a function of time from measured pressures. Such measurements are limited by problems such as the response of the system to pressure changes, variation in the gas temperature within the system, and outgas effects.

Some of the most useful experimental information concerning fission-gas behavior is obtained after the transient. Fuel structure, including swelling, and residual gas concentration are factors that can be studied. The results of such studies can be used to postulate the fission-gas behavior during the transient and the associated posttransient cooling. Interrupted tests are valuable in detailing the transient behavior.

However, it is not feasible to attempt to fully characterize the effects of the many parameters that influence transient fission-gas behavior solely from experiments. A great many tests would be required, and many extreme transient conditions of interest could not be simulated in a prototypic way. It is also difficult in many cases to separate effects of the transient from the effects of the cooldown period.

Thus an analytic approach is needed to provide a comprehensive mechanistic model that includes the effects of major parameters on transient fission-gas behavior. This model must complement the experimental efforts by providing information to aid in the design of critical experiments, and its development must be based on information obtained from experiments. The major benefit of such a model is that it can be applied to conditions not amenable to direct experimental investigation.

A mode1 that suits these requirements has been developed and has shown some capability of predicting behavior compatible with experimental observations. 
The detailed model is embodied in the FRAS (Fission-gas Release and Swelling) computer code, ${ }^{1-3}$ and will be described briefly in Sect. II. The FRAS code is useful for studying the influences of the various parameters on transient gas behavior and for predicting gas behavior in experiments. In addition, general. predictions can be made for arbitrary transients.

However, there are time and space limitations in applying these calculations directly as subordinate calculations in a multi-node computer calculation. A simpler representation is needed to express the primary results of the detailed calculation. Such a representation is developed in parametric form in Sect. III. The results of calculations with the Parametric FRAS (PFRAS) code are compared to corresponding results from the FRAS code for a variety of conditions in Sect. IV. These results show that the PFRAS code provides an excellent approximation to the FRAS results for the spectrum of variables considered. In addition, the results illustrate the dependence of transient gas release and intragranular swelling on the dominant variables. To place the results in proper perspective, however, it is necessary to be familiar with the assumptions used and the limitations inherent in the FRAS calculations. We therefore begin with a discussion of the mechanistic analysis. 


\section{MECHANISTIC ANALYSIS: THE FRAS CODE}

This section has two objectives. First, the general problem of transient fission-gas behavior is discussed briefly so that the problems treated here can be clearly identified. This objective is met by including a discussion of assumptions and limitations of the analysis. Second, the manner in which the detailed analysis is carried out has not been discussed in a comprehensive way; we shall attempt to collect the various bits and pieces here, so that the entire model can be viewed as critically as desired.

Transient fission-gas behavior encompasses a broad range of phenomena, and is influenced by a wide variety of parameters. Composition, including $\mathrm{Pu}, \mathrm{U}, \mathrm{O}$, and impurities, can have significant effects on material properties. Since the influence of these parameters on fission-gas behavior is not at all well characterized, the fuel is assumed to be composed of near-stoichiometric $\left(\mathrm{U}_{0} .{ }_{8} \mathrm{Pu}_{0.2} \mathrm{O}_{2}\right.$. Another important characteristic of as-fabricated fuel is the unrestructured grain size. This is a particularly important parameter for transient fission-gas behavior, because much of the gas retained in irradiated fuel is trapped within the unrestructured fuel grains.

During normal operation, the fuel undergoes several changes that would modify fission-gas behavior in a subsequent transient. Early in life, the fuel restructures; grain growth and porosity migration occur at higher fuel temperatures. Fission gas is generated at a rate dependent on power. Other fission products are also generated, modifying material parameters (including surface tension and the surface-diffusion coefficient). Redistribution of components may occur, varying enrichment and stoichiometry, as we11 as impurity concentrations, across the pin radius. Fission gas may precipitate to form bubbles, but may also be redissolved into supersaturated solution in the fuel by fission fragments (the "re-solution" process). The temperature profile in the pin is determined by the power generated within the pin, the heat removed from its surface, and the temperature-, composition-, and porosity-dependent thermal conductivity of the fuel. The power and cooling histories are therefore important parameters in describing the evolution of irradiated fuel. This evolution is analyzed with computer codes that model fuel behavior during normal reactor operation, such as the LIFE code. ${ }^{4}$

For the purposes of transient analysis, it is assumed that fission gas is retained as a supersaturated solid solution of single gas atoms within spherical 
grains. Variations in composition and material properties are ignored. The effects of dislocations within the grains are ignored, even though they may be important in the early transient behavior of the fission gas.

The transient can be considered as a mismatch between pin power and heat removal capability. The radial temperature profile in the pin may change rapidly. The fuel is subjected to stresses induced by cladding coustraints when the gap closes and to thermal stresses caused by radial variations in thermal expansion. Fission gas causes swelling, which also interacts with the stress field. Fission-gas bubbles also precipitate on grain boundaries. This gas can escape to interlinked porosity at grain edges, or cause swelling and loss of continuity at the grain boundaries. These possibilities also affect the mechanical response of the fuel, as the gas in interlinked porosity can move hydrodynamically down the pressure gradient, or gas on grain boundaries could reduce the tensile strength of the fuel to near zero, greatly enhancing crack propagation. Melting fuel introduces a whole new series of complications, including stress effects due to the volume increase associated with melting.

Many of these considerations are not considered further in the present work, which is intended to describe the intragranular behavior of fission gas during the transient. However, the results described here are prerequisite for further analysis of the behavior of fission gas in fuel pins that fail as a result of a thermal transient. It is assumed that the release of gas from the grains to grain boundaries is the slowest step in gas movement through the pin. The present analysis is therefore aimed at clarifying and quantifying this ratecontrolling step.

The premise of the analysis is that fission gas precipitates rapidly into stable bubbles, that the bubbles are mobile, and that they either coalesce with one another or migrate to grain bcundaries, where they are trapped. The bubbles are assumed to migrate by surface diffusion.

A generalized treatment of the migration and coalescence phenomenon was developed earlier, ${ }^{5}$ based on a number of simplifying assumptions. These assumptions, which are not made in the present analysis, included ideal-gas behavior, small bubbles diffusing according to the same rate laws as larger ones, and no effect of swelling on the coalescence probabilities. The current analysis uses specific properties for mixed-oxide fuel, and carries out a detailed analysis of evolution of the bubble-size probability distribution function 
as it is affected by time, temperature, thermal gradient, and pressure. The analysis corresponds in each case to one representative point, or node, in the fuel pin. Specific areas of analysis will be detailed in the following subsections.

\section{A. Van der Waals Equation of State}

The equation of state for the fission gas is approximated by the modified Van der Waals equation for xenon. The relation used is

$$
P_{g}(V-m b)=m k T \text {, }
$$

where $\mathrm{P}_{\mathrm{g}}$ is the gas pressure within the bubble, $\mathrm{V}$ is the volume of the bubble, $\mathrm{b}=85 \times 10^{-21} \mathrm{~mm}^{3} /$ atom is the Van der Waals coefficient for xenon, ${ }^{6} \mathrm{~m}$ is the number of gas atoms in the bubble, $\mathrm{k}$ is the Boltzmann constant, and $\mathrm{T}$ is the absolute temperature.

The gas pressure is given by the sum of the surface tension restraint of the matrix and the local hydrostatic component of the stress P;

$$
P_{g}=2 \gamma / r+P
$$

where $\gamma$ is the surface tension (taken to be $\left.0.626 \mathrm{~J} / \mathrm{m}^{2}\right)^{7}$ and $r$ is the bubble radius. Substitution for $\mathrm{P}_{\mathrm{g}}$ from Eq. (2) and $\mathrm{f}$ or $\mathrm{V}$ in terms of $\mathrm{r}$ into $\mathrm{Eq}$. (1) results in the following expression for $r$ as a function of $m, P$, and $T$ :

$$
4 \pi \mathrm{Pr}^{4}+8 \pi \gamma \mathrm{r}^{3}-3 \mathrm{~m}(\mathrm{bP}+\mathrm{kT}) \mathrm{r}-6 \gamma \mathrm{mb}=0 .
$$

If $\mathrm{P}=0$, this expression is evaluated directly to determine the bubble radius for each bubble size, characterized by $\mathrm{m}$. For non-zero P, a Newton-Raphson iteration ${ }^{8}$ is used to obtain the desired solution. This calculation is carried out for each size class and for each time step in the analysis, so that the variation in size follows the transient changes in temperature and pressure.

\section{B. Bubble Migration by Surface Diffusion}

A basic premise of the FRAS analysis is that bubbles are mobile under transient conditions. The migration mechanism is assumed to be surface diffusion, which involves migration of atoms over the inner bubble surface and leads 
to a net motion of the center of the bubble. Surface diffusion is thought to occur by an aton, initially attached in the surface, acquiring sufficient energy to becone mobile, after which the aton can move largc distances over the surface before becoming bound. The mean surface-diffusion jump distance ${ }_{s}$ is typically expected to be in the range 100-1000 interatomic distances. ${ }^{9}$

Such large jump distances present a complication in the analysis of bubble diffusion, because the net motion of the diffusing atom may be much less than its jump distance if the bubble is small enough. The results of the analysis 10 of this effect are used in the relations for bubble diffusivity in the FRAS code, and are presented very briefly here.

A straightforward analysis of the random walk of atoms on the interior surface of a bubble leads to the following expression for the bubble diffusivity:

$$
\mathrm{D}_{\mathrm{b}}=\frac{3 \cup \Omega^{2} \mathrm{D} \mathrm{s}}{2 \pi \mathrm{r}^{4}}
$$

Here $\nu$ is the surface density of diffusing atoms (i.e., the rate-controlling specie), $\Omega$ is the molecular volume, and $D_{S}$ is the surface-diffusion coefficient, assumed to be given by an Arrhenius expression $D_{S}=D_{S}^{0} \exp \left(-\Delta H_{S} / R T\right)$. The same result can be obtained by considering the migration of a pore due to the influence of an external force, such as a thermal gradient. 5

Figure 1 illustrates the mean jump distance $\lambda_{S}$ for a pore of radius $r$. It is assumed that $\lambda_{\mathbf{S}}$ is constant and independent of sulface curvature or orientation. Only the resultant vector $\lambda_{s}^{\prime}$, the effective jump distance, contributes to net pore migration. This effective jump distance is given by

$$
\lambda_{s}^{\prime}=2 r \sin \frac{\lambda_{s}}{2 r}
$$

For $r<\lambda_{s} / \pi, \lambda_{s}^{\prime}$ is a periodic function; for this range in $r$, it is assumed that $\lambda_{s}^{\prime}=2 r$.

To correct Eq. (4) for the jump-distance effect, the correction factor $\left(\lambda_{s} / \lambda_{s}\right)^{2}$ must be applied. 10 The resulting relations for bubble diffusivity are 


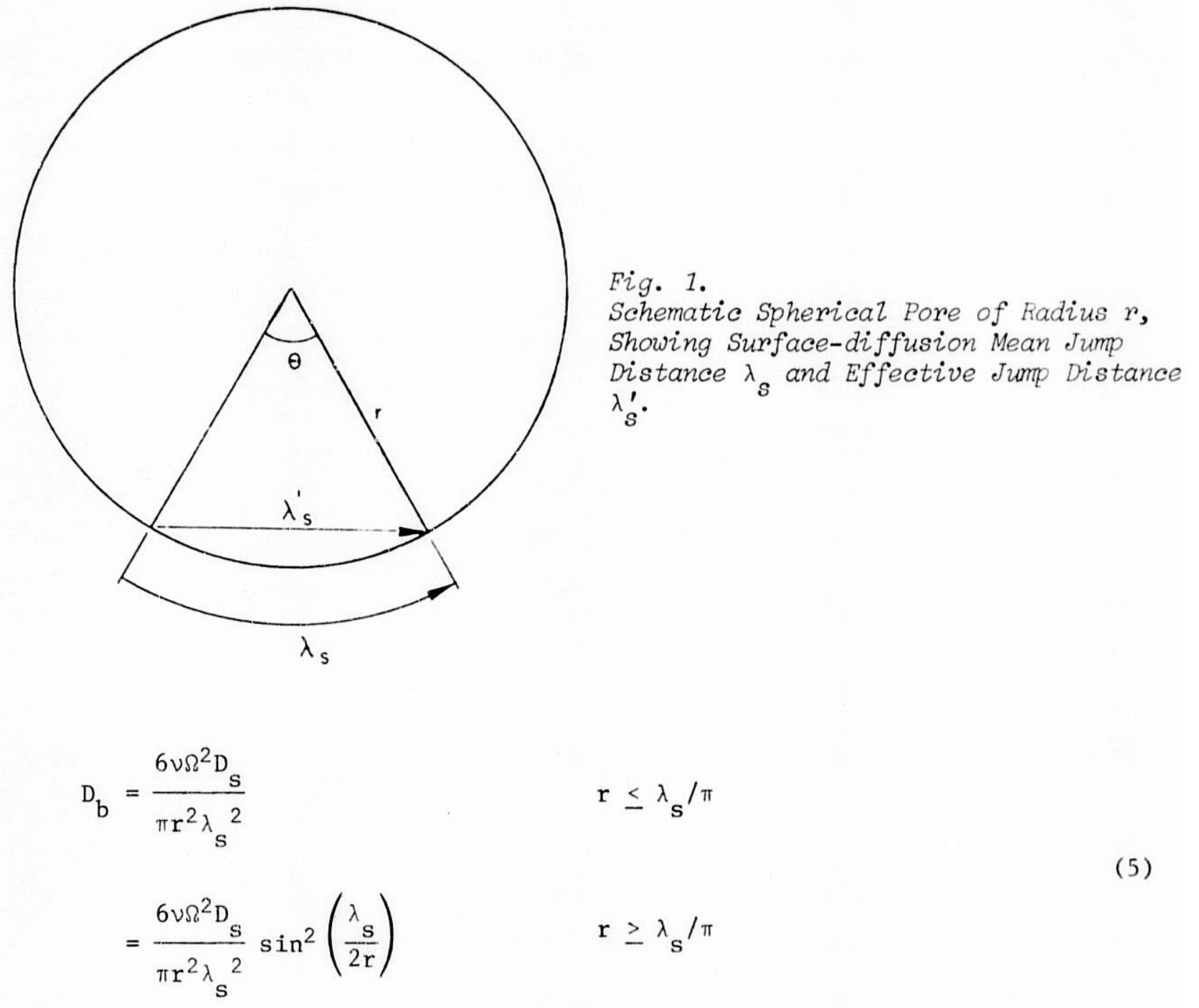

From analysis of observations of bubble migration in irradiated $\mathrm{UO}_{2}, 11$ the mean jump distance was calculated to be about $1.12 \times 10^{-2} \mu \mathrm{m}$. This value is used with Eq. (5) to describe bubble diffusivity in mixed-oxide fuel.

In a thermal gradient, there is a tendency for a bubble to migrate as though it were subjected to a force $F$, given by

$$
\mathrm{F}=-\frac{4}{3} \frac{\pi \mathrm{r}^{3}}{\Omega} \mathrm{f}_{\mathrm{a}}
$$

where $f_{a}$ is a force biasing the migration of surface atoms and arising from the surface-diffusion heat of transport $Q_{S}^{*}, 12$ 


$$
\mathrm{f}_{\mathrm{a}}=-Q * \mathrm{ST}_{\mathrm{s}} / \mathrm{T}
$$

Here $\nabla T_{S}$ is the thermal gradient at the bubble surface, which can be approximated by $3 / 2 \nabla T$ for the case of a non-conducting bubble, where $\nabla T$ is the bulk thermal gradient. 13

The drift velocity of a bubble in a thermal gradient can therefore be defined by the Nernst-Einstein relation as

$$
\mathrm{v}=\frac{\mathrm{D}_{\mathrm{b}}}{\mathrm{kT}} \mathrm{F} \text {. }
$$

Substituting for $\mathrm{D}_{\mathrm{b}}$ and $\mathrm{F}$ from Eqs. (5) and (6), and using Eq. (7), it can be shown that, for small bubbles $\left(r<\lambda_{s} / \pi\right)$,

$$
\mathrm{v}=\frac{8 \nu \Omega \mathrm{D}_{\mathrm{s}} \mathrm{Q}_{\mathrm{s}}^{*} \mathrm{TT}}{\lambda_{\mathrm{s}}{ }^{2} \mathrm{kT}^{2}} \mathrm{r}
$$

For larger bubbles, $\mathrm{r} \geq \lambda_{\mathrm{s}} / \pi$, the velocity is

$$
\mathrm{v}=\frac{8 v \sin \mathrm{s}_{\mathrm{s}} \mathrm{Q}^{*} \mathrm{~T} \mathrm{~T} r}{\lambda_{\mathrm{s}}{ }^{2} \mathrm{kT}^{2}} \sin ^{2}\left(\frac{\lambda_{\mathrm{s}}}{2 \mathrm{r}}\right) .
$$

For even larger bubbles, for which $r \gg \lambda_{s}$, so that $\sin \left(\lambda_{s} / 2 r\right) \cong \lambda_{s} / 2 r$, the drift velocity becomes

$$
\mathrm{v}=\frac{2 \nu \Omega \mathrm{D}_{\mathrm{s}} \mathrm{Q} \mathrm{s}_{\mathrm{s}} \mathrm{T} \mathrm{T}}{\mathrm{rk \textrm {T } ^ { 2 }}}
$$

Thus the drift velocity at first increases with radius, peaking at an intermediate value of the radius $r_{m}=\lambda_{s} / \pi$, then decreases with further increase in r.

\section{Effect of Swelling on Coalescence Probability}

The gas-atom density in the earlier work was assumed not to change with swelling. However, as the bubbles grow larger, the swelling increases the 
initial volume significantly. The result is a net increase in separation between bubble centers within the grains, with a corresponding reduction in coalescence probability, which is derived in this section.

The effect of swelling is accounted for by modifying the coalescence equation, which is of the general form

$$
\Delta g_{i j}=g_{i} g_{j} \Gamma_{i j} \Delta t
$$

where $\Delta g_{i j}$ is the number of coalescences in unit initial volume between $i-$ and $j$-bubbles in a time step $\Delta t, g_{i}$ and $g_{j}$ are the numbers of $i$ - and $j$-bubbles relative to unit initial volume, and $\Gamma_{i j}$ is an interaction function. The interaction function depends on the probability of two bubbles coalescing. The relations used in the FRAS code are those derived previously: ${ }^{5}$

$$
\Gamma_{i j}=4 \pi\left(D_{b}(i)+D_{b}(j)\right)\left(r_{i}+r_{j}\right)
$$

for random migration, and

$$
\Gamma_{i j}=\pi\left(r_{i}+r_{j}\right)^{2}\left(\left|v_{i}-v_{j}\right|\right)
$$

for biased migration.

The system considered as a basis for the calculations of coalescence is unit initial volume of material, containing a fixed number of gas atoms (or a continuously varying number if further gas generation is considered). The modification required for Eq. (10) is therefore to replace the implicit unit volume with the reference volume $\mathrm{V}^{\prime}=1+\Delta \mathrm{V}$, where $\Delta \mathrm{V}$ is the swelling due to fission-gas bubbles. The result is

$$
\frac{\Delta g_{i j}}{1+\Delta V}=\frac{g_{i}}{1+\Delta V} \frac{g_{j}}{1+\Delta V} \Gamma_{i j} \Delta t
$$

or

$$
\Delta g_{i j}=g_{i} g_{j} \Gamma_{i j} \Delta t /(1+\Delta V)
$$


This reduction in coalescence probability is taken into accour.t in the FRAS calculations.

\section{Product-bubble Classification}

It was pointed out in the earlier analysis ${ }^{5}$ that the bubble-size distribution appears to be $\log$ normal for biased-migration coalescence. The distribution for random-migration coalescence is also approximately log normal, although it is not as severely skewed when plotted on a linear basis. In the present analysis, the size classification is based on a logarithmic scale, and coalescence probabilities for random and biased migration are summed.

Some difficulty was encountered in the earlier work in selecting the appropriate size classification $\mathrm{k}$ for the product bubble formed upon coalescence of a bubble from the $i^{\text {th }}$ class with one from the $j^{\text {th }}$ class. This difficulty arises from the approximation of the distribution by discrete class intervals. Selection of $\mathrm{k}$ was made only on the basis of bubble numbers, so that one bubble disappears for each coalescence. The total number of gas atoms, calculated as a check on the accuracy, was found to vary slightly. This limitation has been remedied in the more recent calculations by partitioning product bubbles into two adjacent classes, accounting both for the number of bubbles and for the number of gas atoms. The partitioning is relatively straightforward, and will be described briefly.

If $\Delta g$ bubbles in class $i$ coalesce with $\Delta g$ bubbles in class $j$, then the product bubbles will be distributed into two classes, $k$ and $k+1$, so that

$$
\Delta \mathrm{g}_{\mathrm{k}}+\Delta \mathrm{g}_{\mathrm{k}+1}=\Delta \mathrm{g}
$$

If the number of gas atoms per bubble in an arbitrary class $\ell$ is $m_{\ell}$, then the total number of gas atoms will be unchanged, or

$$
\Delta g_{k} m_{k}+\Delta g_{k+1} m_{k+1}=\Delta g\left(m_{i}+m_{j}\right)
$$

Solving Eqs. (12) and (13) for the two unknowns $\Delta \mathrm{g}_{\mathrm{k}}$ and $\Delta \mathrm{g}_{\mathrm{k}+1}$, we obtain

$$
\Delta g_{k}=\Delta g \frac{m_{k+1}-m_{i}-m_{j}}{m_{k+1}-m_{k}}
$$


and

$$
\Delta \mathrm{g}_{\mathrm{k}+1}=\Delta \mathrm{g}-\Delta \mathrm{g}_{\mathrm{k}}
$$

The class $\mathrm{k}$ is selected to satisfy the requirement

$$
\mathrm{m}_{\mathrm{k}} \leq \mathrm{m}_{\mathrm{i}}+\mathrm{m}_{\mathrm{j}}<\mathrm{m}_{\mathrm{k}+1}
$$

E. FRAS Calculation of the Evolution of the Bubble-size Distribution

Gas release, which depends on bubble mobility, and swelling, which depends on gas volume per gas atom, are both strongly dependent on bubble size. The calculation of the probability distribution function that characterizes the bubble sizes throughout the transient is carried out in the FRAS code. The finite-difference approach used in this calculation is quite similar to that described previous $1 y,{ }^{5}$ except that specific properties for oxide fuel are used, rather than dimensionless parameters, and some of the simplifying assumptions have been removed, as described above.

It was shown in the earlier report ${ }^{5}$ that the bubble-size distributions tend to be log normal. Distribution functions for biased migration are shown in Fig. 2 for the idealized case for two values of the dimensionless time parameter $\tau^{\prime}$. This parameter will be discussed in more detail in Sect. III. The disappearance of bubbles by coalescence and the tendency toward increasing bubble sizes with increasing time are illustrated.

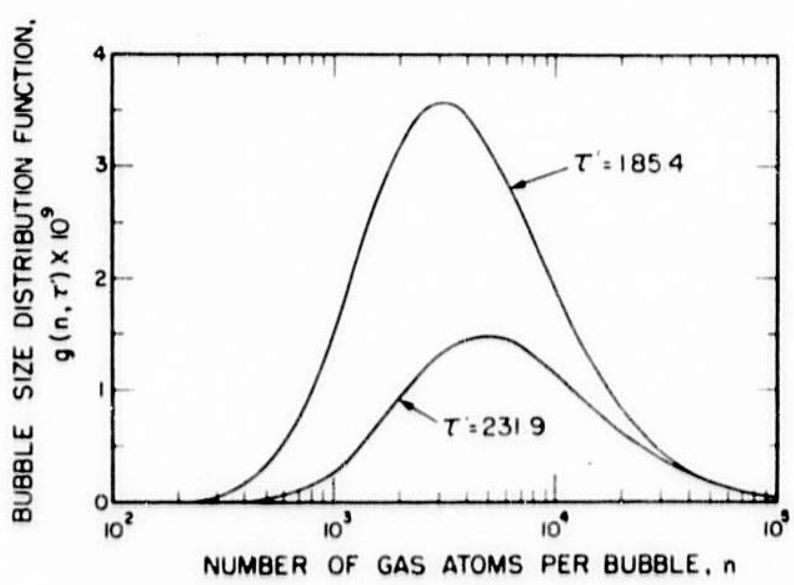

Fig. 2. Bubble-size Distribution Functions for Idealized Conditions (from Ref. 5).

A more specific case is illustrated in Fig. 3, which shows the response of fission gas in oxide fuel to a thermal transient intended to simulate a loss of coolant event without reactor scram. The fuel is heated almost to melting 


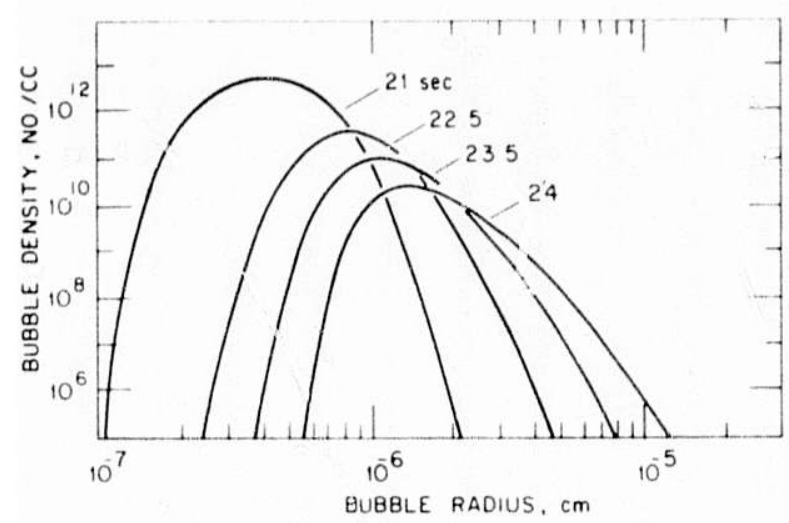

Fig. 3.

Evolution of Bubble-size Distribution

Function for Oxide Euel Subjected to

a Simulated Thermal Transient.

at $24 \mathrm{~s}$ into the transient. These results were calculated with the current FRAS code and incorporate the considerations outlined above, as well as the fact that random and biased migration occur concurrently. The results again show the disappearance of small bubbles as they coalesce, increasing the mean bubble size.

These distribution functions are calculated in FRAS as functions of the transient history, taking into account variations in the temperature, thermal gradient, and pressure as functions of the transient time. The results are used to calculate the total bubble volume, which gives the intragranular swelling, and the bubble mobility, which is used to calculate the gas release.

\section{F. Gas Release from Grains to Grain Boundaries}

Gas release is assumed to occur by both random and biased migration of bubbles. A simplified approach, based on a mean mobility of gas atoms, is used in the present ana1vsis.

Random-migratio release of gas bubbles from a spherical grain is calculated from the relation given by $\mathrm{Crank}^{14}$ for diffusion of solute from a sphere. The fractional gas release $\mathrm{f}_{\mathrm{r}}$ is

$$
f_{r}=1-\frac{6}{\pi^{2}} \sum_{n=1}^{\infty} \frac{1}{n^{2}} \exp \left(-n^{2} \pi^{2} D t / a^{2}\right)
$$

where $a$ is the grain radius and $D$ is the bubble diffusivity. To apply this result to the transient, the product Dt is determined as the integral of the mean bubble diffusivity over the transient. The mean diffusivity in turn is determined from the bubble-size distribution and transient conditions at each time step. 
Since migration distance is the important factor, $D$ is defined from the mean value of $\sqrt{D_{b}}$. The mean is also weighted according to the number of gas atoms per bubble, to give the best estimate of gas released. The result can be expressed by

$$
D t=\int_{0}^{t}\left(\frac{\sum_{i=1}^{N} \sqrt{D_{b}^{(i)}} m_{i} g_{i}}{\sum_{i=1}^{N} m_{i} g_{i}}\right)^{2} d t^{\prime},
$$

where $\mathrm{N}$ is the number of size classes used to define the bubble-size distribution.

The difference between this approach and the much earlier Booth model $1^{15}$ is in the variation of $\mathrm{D}$, which changes with the evolution of the bubble-size distribution as well as with temperature. For small values of Dt, the first few terms in the series of Eq. (17) are not sufficient, and the approximation due to J. M. Kennedy, as referenced by Booth, ${ }^{15}$ is used. This approximation, which can be written

$$
\mathrm{f}_{\mathrm{r}} \cong 6\left(\mathrm{Dt} / \mathrm{a}^{2} \pi\right)^{\frac{1}{2}}-3 \mathrm{Dt} / \mathrm{a}^{2}
$$

is sufficiently accurate for release up to about $90 \%$.

Biased-migration release is calculated with the approximation that all of the bubbles move uniformly across the grain with the same velocity $\bar{v}$ until they are trapped at the boundary. As in the calculation of release by random migration, the bubble mobility is weighted according to the number of gas atoms per bubble. The mean bubble velocity is defined by

$$
\bar{v}=\frac{\sum_{i=1}^{N} v_{i} m_{i} g_{i}}{\sum_{i=1}^{N} m_{i} g_{i}},
$$


where $v_{i}$ is calculated according to Eqs. (9a) and (9b) for each size class and each time step.

The result is applied in a simple geometric model that describes the motion of one sphere, a conceptual envelope for the gas, relative to a stationary one, the spherical grain. The overlap of these two spheres, illustrated in Fig. 4,

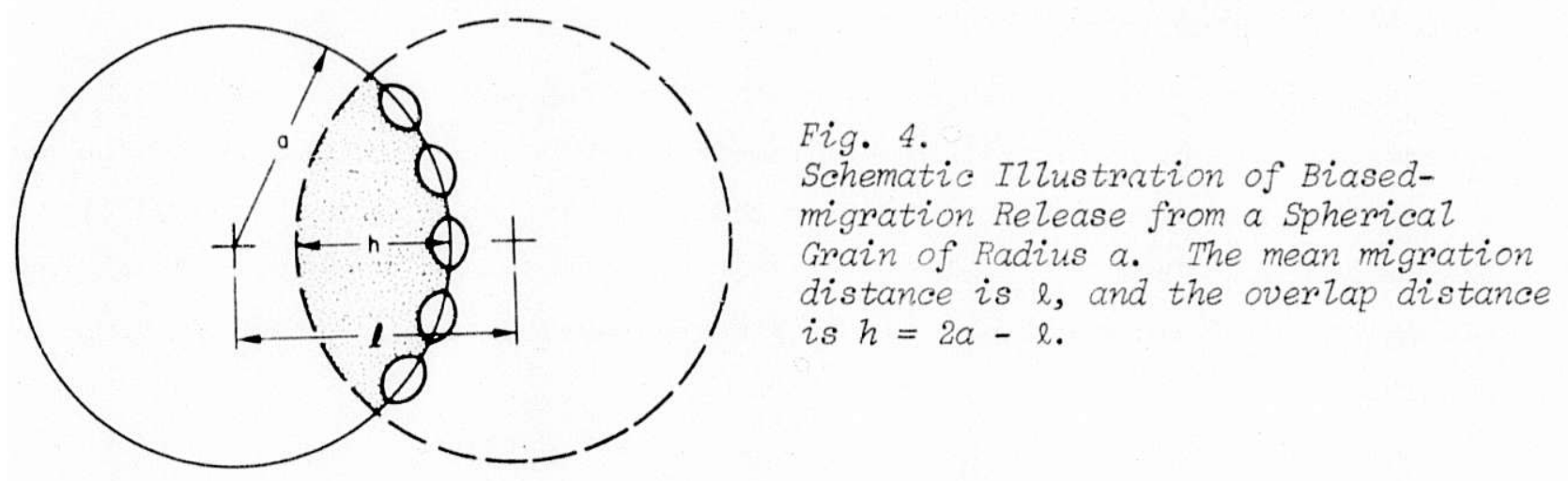

represents the volume fraction of the grain that still retains gas at the assumed original density; the remainder of the grain is assumed to have released a11 of the gas. This model is applied by integrating the mean velocity over the transient time to obtain the cumulative migration distance. Subtracting this distance from the grain diameter gives the overlap distance $h$, from which the gas release fraction by biased migration is obtained by simple geometry in the form 16

$$
f_{b}=\frac{h^{2}(3 a-h / 2)}{8 a^{3}}
$$

The release fractions $\mathrm{f}_{\mathrm{r}}$ and $\mathrm{f}_{\mathrm{b}}$ calculated according to Eqs. (19) and (21) must be combined in a consistent way to give the overall release fraction. Preliminary calculations ${ }^{1}$ have indicated that random-migration release is generally smaller and occurs earlier in the transient than biased-migration release. Random migration is therefore expected to promote uniform release from the regions adjacent to grain boundaries. When biased migration becomes significant, the first gas that would be released is that at the leading edge of the moving envelope of gas bubbles. However, random migration has already caused some of this gas to be released.

To avoid double counting of the release, the release due to biased migration is not considered until it exceeds half of the random release. The other 
half of the random release would reduce the late release due to biased migration, and is accounted for simply by not allowing release to exceed $100 \%$. The total release $\mathrm{f}$ is therefore defined as the sum of random-migration release and the portion of biased-migration release that is not redundant, or that exceeds half the random-migration release.

\section{G. Intragranular Swe1ling}

The swelling is calculated very simply as the sum of the bubble volume associated with unit initial fuel volume. However, swelling is assumed to occur only in the portion of the grain that contains fission gas, with no swelling in the remainder. Thus the swelling is corrected for gas release by multiplying the calculated volume change, assuming no release, by the fraction of gas retained, or

$$
\Delta V^{\prime}=(1-f) \sum_{i=1}^{N} V_{i} g_{i}=(1-f) \Delta V
$$

where $V_{i}$ is the volume of a bubble in class $i$ and $\Delta V$ is the fractional swelling in that portion of the grain that retains fission gas.

The intragranular swelling calculated here is only one component of fuel swelling; the subsequent effects of released gas on the fuel microstructure must be considered in assessing overall swelling. Unless the gas can escape rapidly through interconnected porosity, it may be expected that grain-boundary and grain-edge swelling will be significant.

Another factor that must be considered in swelling predictions is that the coalescence models assume bubhles to be isolated, and that coalescence occurs immediately upon contact of two bubbles. The swelling analysis is only appropriate in the low-swelling regime ( $\Delta \mathrm{V} \ll 30 \%$ or so). Larger values of predicted swelling indicate the likelihood of gross swelling, but should not be considered quantitatively. Gas release results are also subject to this qualification.

Finally, the time required for product bubbles to reach equilibrium size is ignored. This time would be a more important factor for larger bubbles than for small ones. A treatment of this problem, however, is beyond the scope of the present work. 


\section{H. Selection of Material Parameters}

Direct experimental results are not available to specify $Q_{\mathrm{s}}^{*}$, and observations of bubble mobility indicate that measured values of $D_{s}$ overpredict bubble mobility by ordars of magnitude. ${ }^{9}$ Some comment on the values being used in the analysis is therefore necessary.

The heat of transport $Q_{S}^{*}$ is taken to be $67 \mathrm{kcal} / \mathrm{mole}^{*}$ The results are not too sensitive to the choice of this parameter, because it occurs always as a product with $D_{S}$ (see Eq. (9a), for example). The magnitude of $Q_{S}^{*}$ therefore has the effect of determining the relative importance of random and biased migration. If the selected value is too large, future measurements will show a larger amount of random-migration release than currently predicted.

The surface-diffusion coefficient was selected to be compatible with the limited data available for transient gas release. ${ }^{1}$ The expression used is

$$
D_{s}=3.5 \times 10^{4} \exp \{-90 / R T\}
$$

where RT has dimensions $\mathrm{kcal} / \mathrm{mole}$.

These parameters are expect. 1 to be modified as the experimental data base, which is at present extremely limited, improves.

\footnotetext{
The value $100 \mathrm{kcal} / \mathrm{mole}$ was originally assumed, but an oversight in the calculation lead to omission of the $3 / 2$ factor relating the bubble-surface thermal gradient to the bulk thermal gradient. Decreasing the value of $Q_{\mathrm{s}}^{*}$ retains the validity of the original calibration of the FRAS code.
} 


\section{PARAMETRIC SYNTHESIS OF FRAS RESULTS: PFRAS}

The FRAS code is relatively fast and convenient for single-node analysis of transient fuel behavior, but the detailed calculations described in Sect. II make it time consuming and somewhat cumbersome for multi-node analyses. It would be necessary to devote considerable computer storage to the various arrays describing the bubble-size distributions at each node.

Considerable effort has therefore been directed to the development of simpler methods to synthesize the results that would be obtained from FRAS calculations. Some success was achieved by developing a relatively simple analytical expression for the gas release rate. The parameters in this expression were determined by comparison to the results of a series of FRAS calculations. In one application of this approach, results were presented for one grain size, for atmospheric pressure, and for a limited range in initial gas concentration. ${ }^{17}$ This approach was later extended to define parameters appropriate for a larger grain size, a broader range in initial gas concentration, and for the higher pressure $(8.6 \mathrm{MPa}$, or $85 \mathrm{~atm})$ typical of the GCFR. ${ }^{18}$

Some difficulties were encountered in applying this approach. Many FRAS calculations had to be carried out, and the results had to be laboriously converted to suitable parameters. Even then, the results were limited in their range of application. Some effects, including swelling and varying hydrostatic pressure, could not be incorporated into the formalism.

An alternative approach, based on analysis of coalescence in terms of a dimensionless time, has therefore been used to develop a much more general algorithm. This algorithm, which is the basis of the Parametric FRAS (PFRAS) code, constitutes a more direct analysis of fission-gas behavior than the simpler method. Only two parameters are required in this algorithm (one for gas release, the other for swelling). Tabulated values of these parameters are initially determined from results of FRAS calculations. The values used in subsequent PFRAS calculations are obtained by two-dimensional interpolation, which introduces some additional complexity when compared to application of the earlier method.

This additional complexity is greatly outweighed by the advantages of the algorithm used in the PFRAS code. The fuel-grain size is included explicitly, so an arbitrary grain size can be considered for each fuel node. Local 
hydrostatic pressure is included as an explicit transient variable, and intragranular swelling is calculated; the interaction of swelling with the local stress can therefore be included in pin-mechanics modeling. Only eight FRAS calculations (one for each pressure considered) were required to derive the tables of parameters used in the PFRAS code. Finally, the PFRAS code provides results that accurately approximate FRAS results over the entire range of validity of the FRAS code.

Fundamental to the generalized parametric analysis is the dimensionless time, first defined to simplify the original analysis. ${ }^{5}$ Preliminary calculations with the FRAS code have shown that biased migration is the dominant process to be considered. The dimensionless time from the original analysis for biased migration can be written as

$$
\tau^{\prime}=\left(\frac{3 \pi}{2 \gamma \mathrm{k}}\right)^{\frac{1}{2}} \Omega \nu Q_{S}^{*} \mathrm{C}_{\mathrm{o}} \mathrm{D}_{\mathrm{s}} \mathrm{t} \nabla \mathrm{T}_{\mathrm{s}} / \mathrm{T}^{3 / 2},
$$

where $\mathrm{C}_{o}$ is the pretransient fission-gas concentration, and the other parameters were defined in the preceding section. Equation (24) was derived for steadystate applications (in particular, for postirrarliation annealing). The effect of local swelling on coalescence probabilities was ignored, but can be included in the definition of dimensionless time by dividing by $(1+\Delta V)$, as indicated by Eq. (11). Equation (24) can then be generalized to transient conditions by inc.luding the time-dependent terns in an integral; the generalized definition of $\tau$ becomes

$$
\tau=\left(\frac{3 \pi}{2 \gamma k}\right)^{\frac{1}{2}} \Omega V Q_{S}^{*} C_{0} \int_{0}^{t} \frac{D_{s} \nabla T s}{T^{3 / 2}(1+\Delta V)} d t^{\prime} .
$$

The bubble-size distribution function $G$ changes in a characteristic way with the dimensionless time, such that a normalized form tends to become invariant with further changes in time. It has been shown ${ }^{5}$ that a consistent definition of the distribution function is

$$
G(m, t)=C_{0} \tau^{-2 p} z(u)
$$


where $\mathrm{Z}(\mathrm{u})$ is the asymptotic time-invariant function, $\mathrm{u}=\mathrm{m} \tau^{-\mathrm{p}}$, and $\mathrm{p}$ is a constant. The results for the simplified analysis for biased migration alone are shown in Fig. 5, which illustrates the tendency of the curves toward an asymptotic form. ${ }^{5}$ For this case, $p=2$.

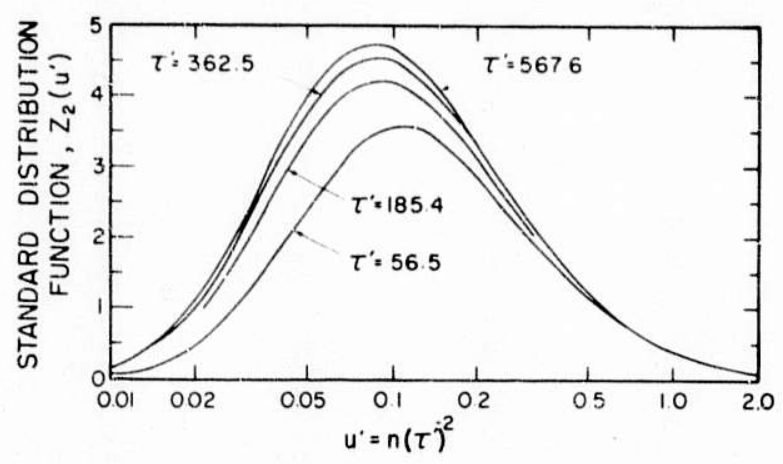

Fig. 5. Variation in the Standard Distribution Function $\mathrm{Z}_{2}$ with Dimensionless time $\tau$ ', Showing the T'endency Toward an Asymptotic Log-normal curve.

The gas release to grain boundaries is calculated from the mean bubble velocity $\bar{v}$ as defined by Eq. (20). Equation (9c) can be used to relate the bubble velocity to its radius, and a simplified form of Eq. (3) (i.e., idea1 gas approximation with $\mathrm{P}=0$ ) can be used to relate $\mathrm{r}$ to $\mathrm{m}$, the number of gas atoms per bubble. The bubble velocity $\mathbf{v}_{\mathbf{i}}$ can therefore be approximated by

$$
\mathrm{v}_{i}=\mathrm{Am}_{i}^{-\frac{1}{2}}
$$

where

$$
A=\frac{2 \nu \Omega D_{s} Q_{S}^{* \nabla T} s}{k T^{2}}\left(\frac{8 \pi \gamma}{3 k T}\right)^{\frac{1}{2}}
$$

Equation (20) can be simplified to

$$
\bar{v}=A \frac{\left\langle m^{1 / 2}>\right.}{<m>},
$$

where $\left\langle\mathrm{m}^{\mathrm{q}}>\right.$ is defined as the $\mathrm{q}^{\text {th }}$ moment of the distribution function $\mathrm{G}$.

From Eq. (26) and the fact that the first moment $<\mathrm{m}>$ is equal to the initial concentration $C_{0}$, the moments can be expressed in terms of moments of the time-invariant distribution function $\mathrm{Z}(\mathrm{u})$ as

$$
<\mathrm{m}^{\mathrm{q}}>=\mathrm{C}_{\mathrm{o}} \tau^{2 \mathrm{q}-2}<\mathrm{u}^{\mathrm{q}}>
$$


The expression for the mean velocity becomes

$$
\bar{v}=A \tau^{-1} \frac{\left\langle u^{\frac{1}{2}}\right\rangle}{\langle u\rangle} .
$$

A varies with instantaneous conditions related to temperature and thermal gradient, as shown in the definition at Eq. (27). These parameters can be rearranged to define the new "constant" $\mu$ as

$$
\mu \equiv \frac{\mathrm{T}^{5 / 2}}{\mathrm{D}_{\mathrm{s}} \nabla \mathrm{T}_{\mathrm{s}}} \mathrm{A} \frac{\left.\mathrm{u}^{\frac{1}{2}}\right\rangle}{\langle\mathrm{u}\rangle} .
$$

Substituting for A in Eq. (30) from Eq. (31) gives the result

$$
\bar{v}=\mu \frac{\mathrm{D}_{\mathrm{S}} \nabla \mathrm{T}}{\mathrm{T}^{5 / 2}} .
$$

Although $\mu$ as defined by Eq. (31) and the definition of A, Eq. (27), appears to be a constant, relaxing the approximations used in its definition shows that it varies with $\tau$. The assumptions of ideal-gas behavior and the approximation of large bubbles, implied in the use of Eq. (9c) - along with the neglect of random-migration considerations and the assumption that the bubblesize distribution has reached the asymptotic form - imply that in fact $\mu$ will be a fairly complex function of $\tau$. Equation (32) is therefore used as the working definition of $\mu$, rather than Eq. (31).

Equation (32) was used in conjunction with the FRAS code to obtain $\mu$ as a function of $\tau$ for a broad range of conditions. Some results are shown in Fig. 6 for zero hydrostatic pressure. A base case was selected to show the results for a constant-temperature anneal at $2200^{\circ} \mathrm{C}$, with $\nabla \mathrm{T}=500^{\circ} \mathrm{C} / \mathrm{mm}$ and $\mathrm{C}_{\mathrm{O}}=2 \times 10^{17}$ gas atoms $/ \mathrm{mm}^{3}$. The temperature, thermal gradient, and initial concentration were varied in both directions from the base case for additional runs. The results show that one curve provides an excellent representation of all of the data.

The effect of pressure must also be considered. Hydrostatic pressure reduces the size of the bubbles, with a greater effect for larger bubbles than for small ones. The bubble-size distribution is therefore shifted toward smaller 


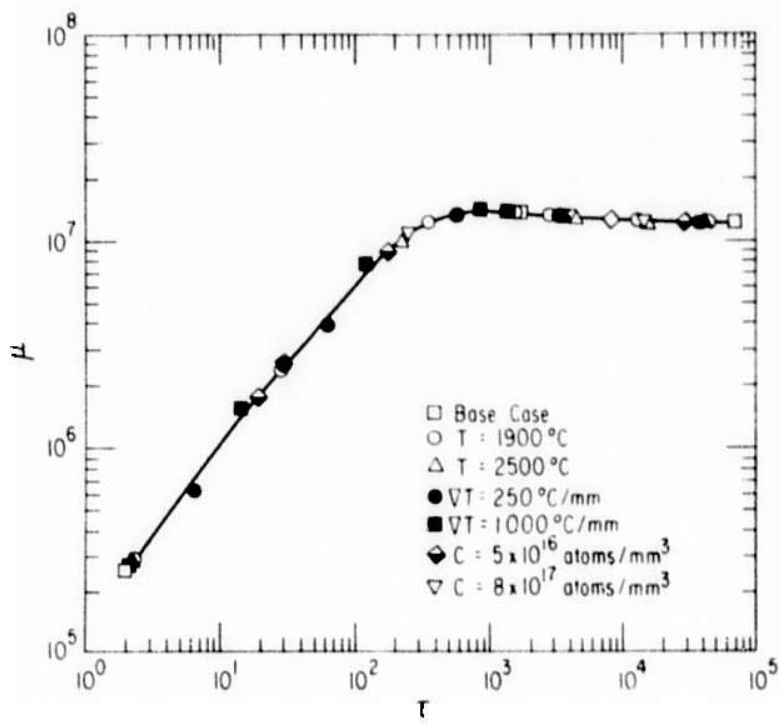

Fig. 6.

Variation of $\mu$ wit $\tau$ at $P=0$ for Various Conditions, as Calculated with the FRAS Code.

sizes at the high end, with little effect at the low end except at very high pressures. It is interesting to note, in the results from the original analysis ${ }^{5}$, that this causes the shape of the distribution function to change toward greater similarity with the random-migration distribution function.

The effects of increasing pressure are illustrated in Fig. 7, which shows the results for a $300^{\circ} \mathrm{C} / \mathrm{s}$ heating ramp, followed by holding at the solidus for

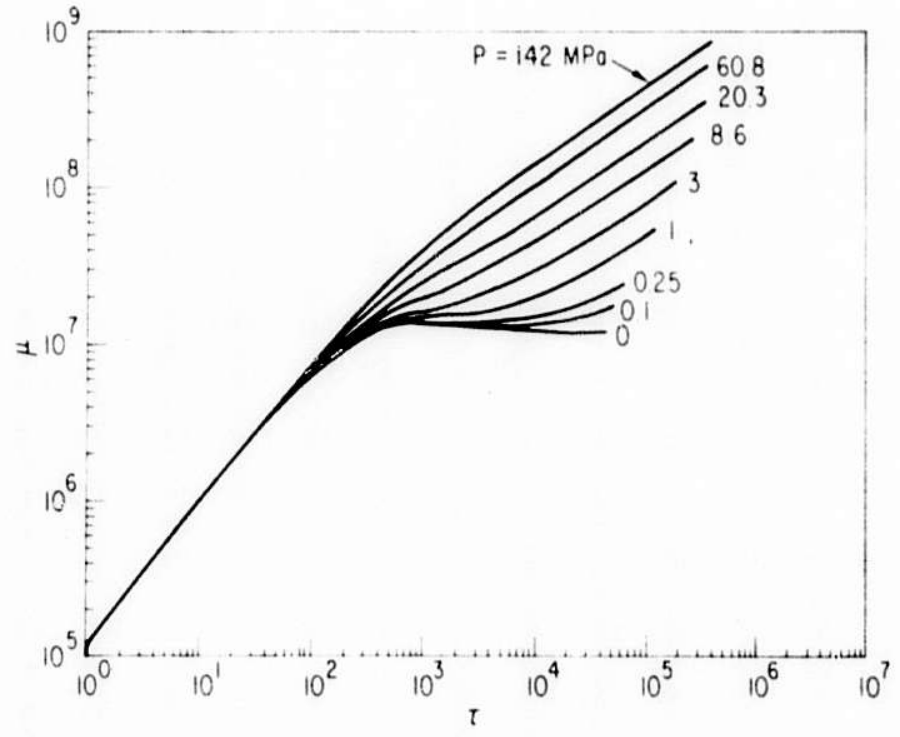

Fig. 7 .

Effect of Pressure on the Variation of $\mu$ with $\tau$.
$5 \mathrm{sec}$ to extend the total range in $\tau$. Initial concentration is $\mathrm{C}_{0}=$ $1 \times 10^{17}$ atoms $/ \mathrm{mm}^{3}$, and a constant thermal gradient of $500^{\circ} \mathrm{C} / \mathrm{mm}$ is assumed. The pressure was held constant at values ranging from 0 to $141.86 \mathrm{MPa}$ (0 to $1400 \mathrm{~atm}$ ), as shown in the figure. The results can be explained qualitatively as described above. Small pressures have little influence until larger bubbles are formed, at larger values of $\tau$. Higher pressures affect smaller bubbles as we11, and the influence extends to smaller values of $\tau$ as the pressure increases. 
The results show the unique dependence of $\mu$ on $\tau$ (which represents all history effects) and on pressure. From this result, Eq. (32) can be used to determine the mobility, which in turn gives the gas-release fraction as described in Sect. II.F above. However, $\tau$ depends on swelling, as shown by Ẽq. (25). Thus, it is necessary (as well as desirable) to calculate the swelling in conjunction with the gas release in this parametric analysis.

The swelling can be calculated in a manner analogous to that used for mean bubble velocity. In terms of the distribution function, the local intragranular swelling $\Delta \mathrm{V}$ is

$$
\Delta V=\frac{4 \pi}{3} \sum_{i=1}^{N} r_{i}{ }^{3} g_{i} .
$$

Again, the same simplifying assumption leads to the approximation

$$
\mathrm{r}=\mathrm{Bm}^{\frac{1}{2}}
$$

where $B=(3 \mathrm{kT} / 8 \pi \gamma)^{\frac{1}{2}}$. In terms of the time-invariant moments, the swelling is

$$
\Delta V=\frac{4}{3} \pi B^{3}\left\langle m^{3 / 2}\right\rangle=\frac{4}{3} \pi B^{3}\left\langle u^{3 / 2}\right\rangle C_{0} \tau
$$

Again $\tau$ accourits satisfactorily for transient history effects, but instantaneous conditions (particularly temperature) affect the swelling. We postpone consideration of the effects of pressure. Rearranging terms and substituting for $B$, we define a new "constant" $\phi$ by the relation

$$
\Delta \mathrm{V}=\phi \mathrm{T}^{3 / 2} \mathrm{C}_{\mathrm{o}} \tau
$$

where

$$
\phi=\frac{4 \pi}{3}\left(\frac{3 k}{8 \pi \gamma}\right)^{3 / 2}\left\langle u^{3 / 2}\right\rangle .
$$

As in the case of the parameter $\mu, \phi$ is actually a variable because the simplifying assumptions do not apply in the detailed analysis. The variation 
of $\phi$ is determined from FRAS calculations, using Eq. (36) as the definition of $\phi$. Figure 8 shows the results for the FRAS calculations discussed in connection with Fig. 6. Again, the results show excellent agreement for a variety of conditions.

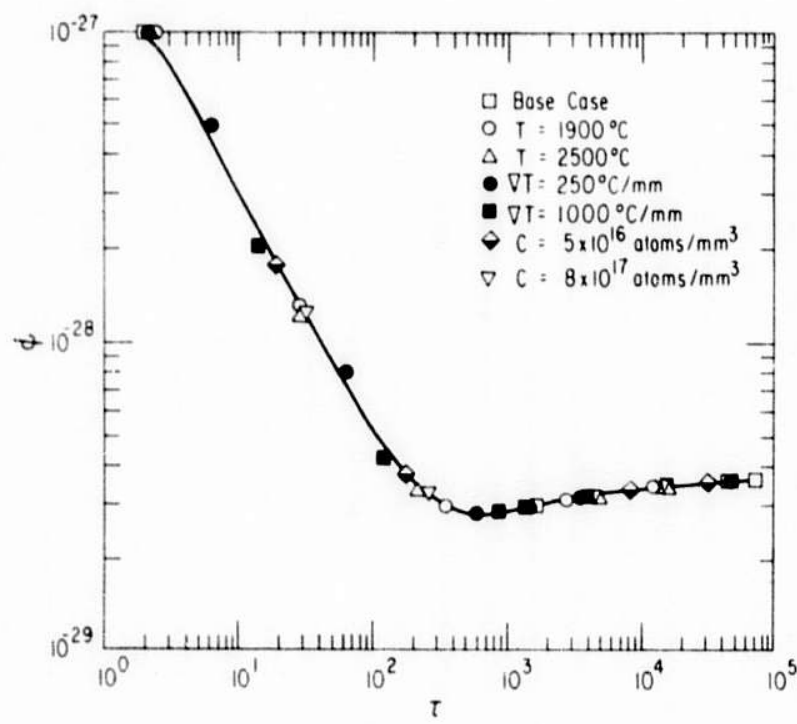

Fig. 8. Variation of $\phi$ with $\tau$ at $P=0$ for various Conditions.

Extending the analysis to a series of pressures, as done for $\mu$ above, results in the family of curves illustrated in Fig. 9. The FRAS calculations

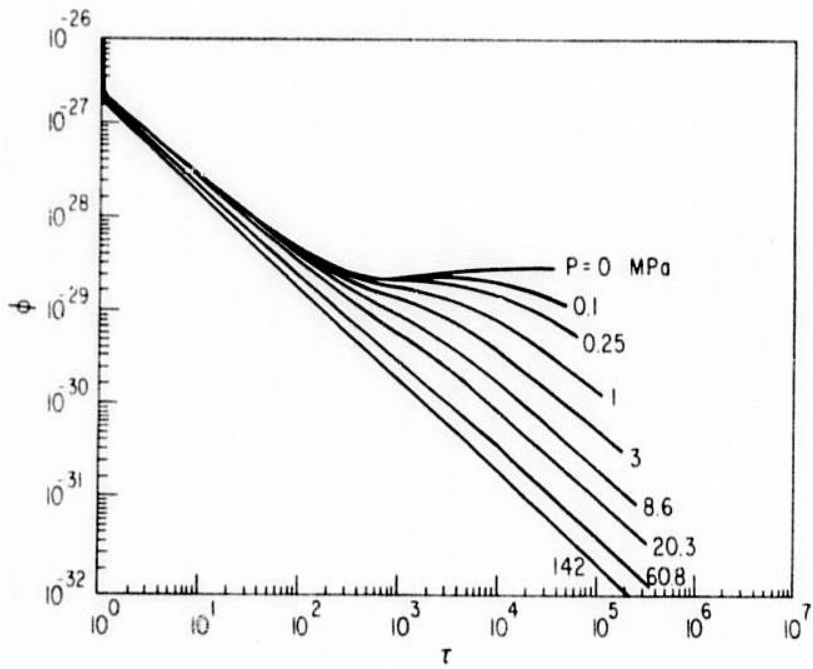

Fig. 9 .

Effect of Pressure on the Variation of $\phi$ with $\tau$.

used in Fig. 7 are applied here. Increasing pressure reduces swelling, thus reducing $\phi$; again, larger bubbles are affected more than smaller ones.

The curves for $\mu$ and $\phi$ are applied to transient analysis of gas release and swelling by applying Eqs. (25), (32), and (36) in an iterative scheme, using a two-dimensional linear interpolation of the FRAS results to determine $\mu$ and $\phi$ for arbitrary $\tau$ and $P$. This approach appears to be the simplest way to apply 
the results. However, it is necessary to verify that the approach provides a good approximation to FRAS results if the pressure varies during the transient.

Figure 10 shows the variation of $\mu$ and $\phi$ with $\tau$ for an abrupt change in pressure during a transient history. The initial gas concentration was

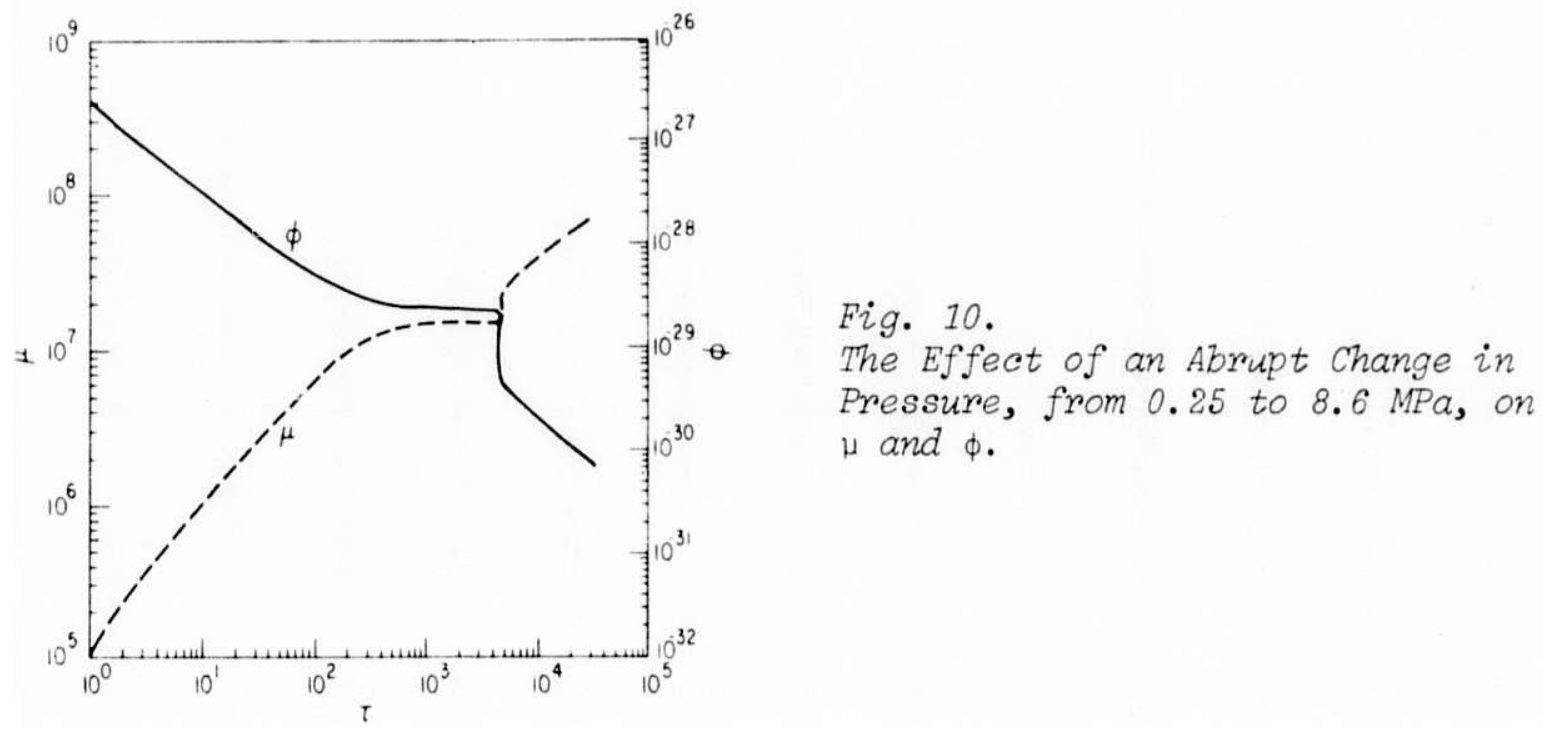

$1 \times 10^{17}$ atoms $/ \mathrm{mm}^{3}$, and the fuel node was assumed to undergo a $300^{\circ} \mathrm{C} / \mathrm{s}$ heating ramp with a constant thermal gradient of $500^{\circ} \mathrm{C} / \mathrm{mm}$. The pressure was held at $0.25 \mathrm{MPa}(2.5 \mathrm{~atm})$ until the temperature reached $2467^{\circ} \mathrm{C}$, when it was increased to $8.61 \mathrm{MPa}(85 \mathrm{~atm})$. For the generalized parametric model to be completely valid, Fig. 10 should indicate instantaneous transitions from the $0.25 \mathrm{MPa}$ curves to the $8.61 \mathrm{MPa}$ curves shown in Figs. 7 and 9 . The transition illustrated in Fig. 10 is more gradual, as should be expected. However, the transition is sufficiently rapid to generate confidence that the generalized parametric approach is a good approximation to FRAS results even for varying pressure histories. 


\section{RESUL.TS}

A Fortran code was written to apply this approach to various initial and transient conditions for comparison to parallel FRAS calculations. This code, called PFRAS (Parametric Fission-gas Release and Swelling), was written as a flexible, stand-alone code, so that arbitrary transients could be considered.

The primary calculation in the code is the determination of the dimensionless time $\tau$. The parameters $\mu$ and $\phi$ are determined from $\tau$ and $P$ by a 1 inear two-dimensional interpolation, on logarithmic scales, of the data illustrated in Figs. 7 and 9. The interpolation procedure is described in the Appendix.

The gas-release fraction is calculated explicitly as a function of grain size from the mobility, as described in Sect. II.F. The sweliing is calculated according to Eq. (36), and enters recursively in the integration of $\tau$ as shown in $\mathrm{Eq} .(25)$.

A number of exploratory transients were analyzed with both the FRAS and the PFRAS codes. The results are shown in Figs. 11-15. In each figure, the results for a base case calculation are repeated, and the parameter of interest is varied from the base value. The lines correspond to FRAS results, and the symbols correspon' to PFRAS results. The base values are $300^{\circ} \mathrm{C} / \mathrm{s}$ heating rate (varied from 100 to 1000 ); $500^{\circ} \mathrm{C} / \mathrm{mm}$ thermal gradient (varied from 100 to 2500); $10 \mu \mathrm{m}$ grain diameter (varied from 4 to 25 ); $1 \times 10^{17}$ gas atoms $/ \mathrm{mm}^{3}$ (varied from 0.2 to $4 \times 10^{17}$ ); and $0.25 \mathrm{MPa}$ pressure (varied to 8.61 and 141.86 ).

The results shown in Figs. 11-15 are included both to show the sensitivity of transient fission-gas behavior to the parameters considered and to demonstrate the applicability of the PFRAS generalized parametric code. Although the swelling is shown to large values in some cases, it should be recalled that swelling greater than about $30 \%$ cannot be predicted accurately (see Sect. II.G). The large predicted values are shown only to indicate the degree of agreement between the PFRAS and FRAS results. In both cases, large predicted values of swelling indicate that gross swelling may be expected, but the calculations should not be interpreted quantitatively.

Figures 11 and 12 show the effects of initial grain size and initial gas concentration on release and swelling. Figures 13, 14, and 15 show the effects

of heating rate, thermal gradient, and pressure. In all cases, the agreement is acceptable. The greatest error occurs for the extreme thermal gradient of 

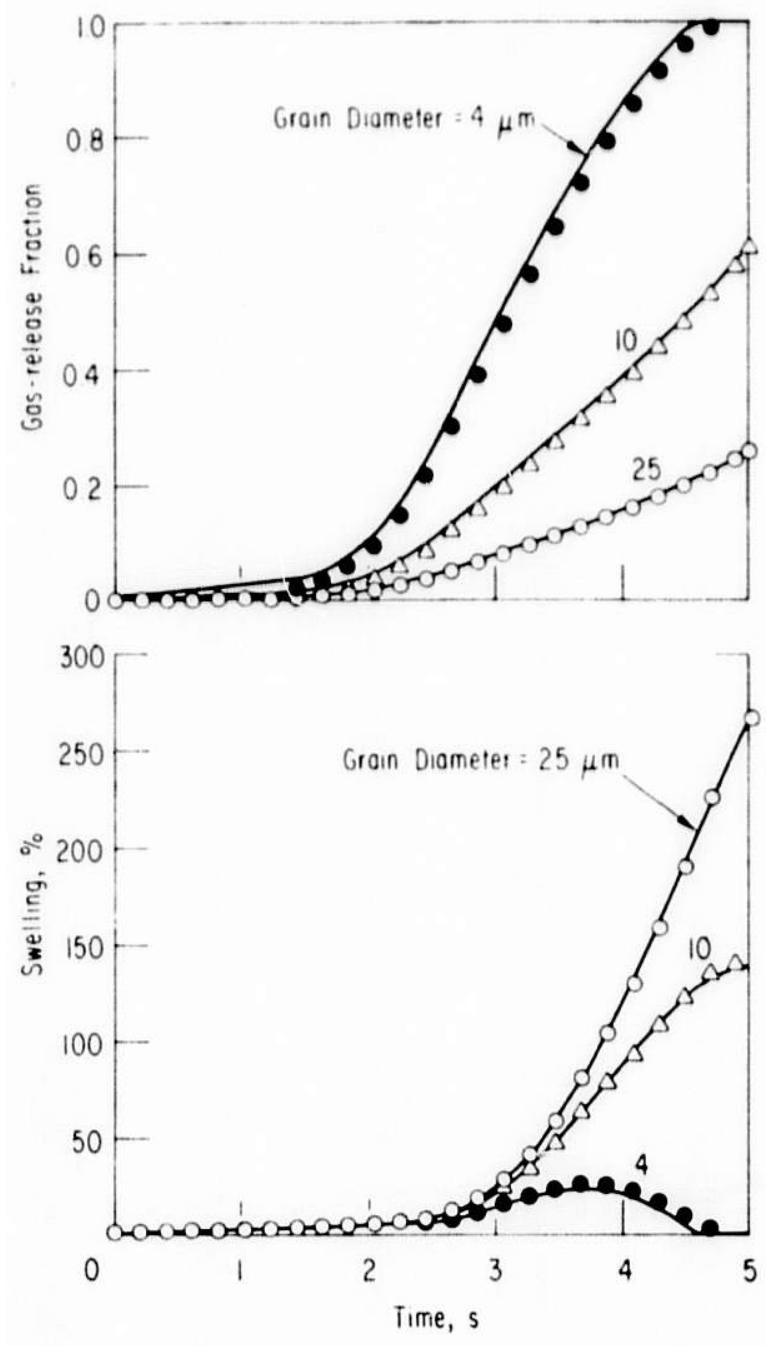

Fig. 11 .

Effect of Grain Size on Gas Release and sweiling. Lines show FRAS results and symbols show PFRAS results.

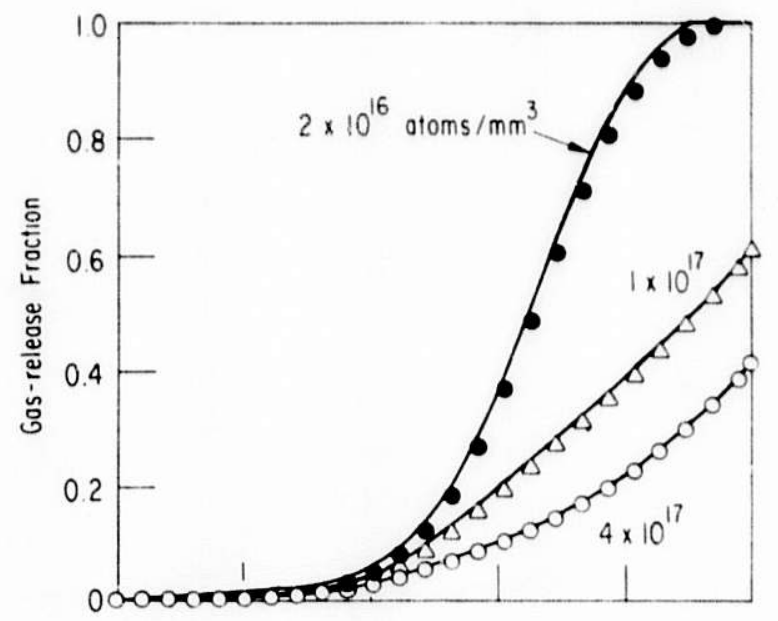

Fig. 12.

Effect of Initial Fission-gas concentration on Gas Release and Swelling.

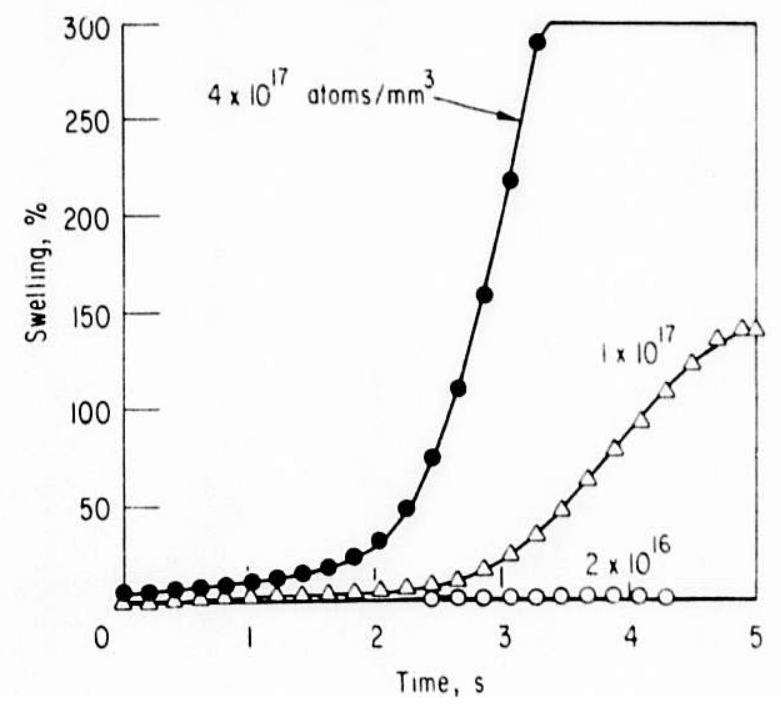



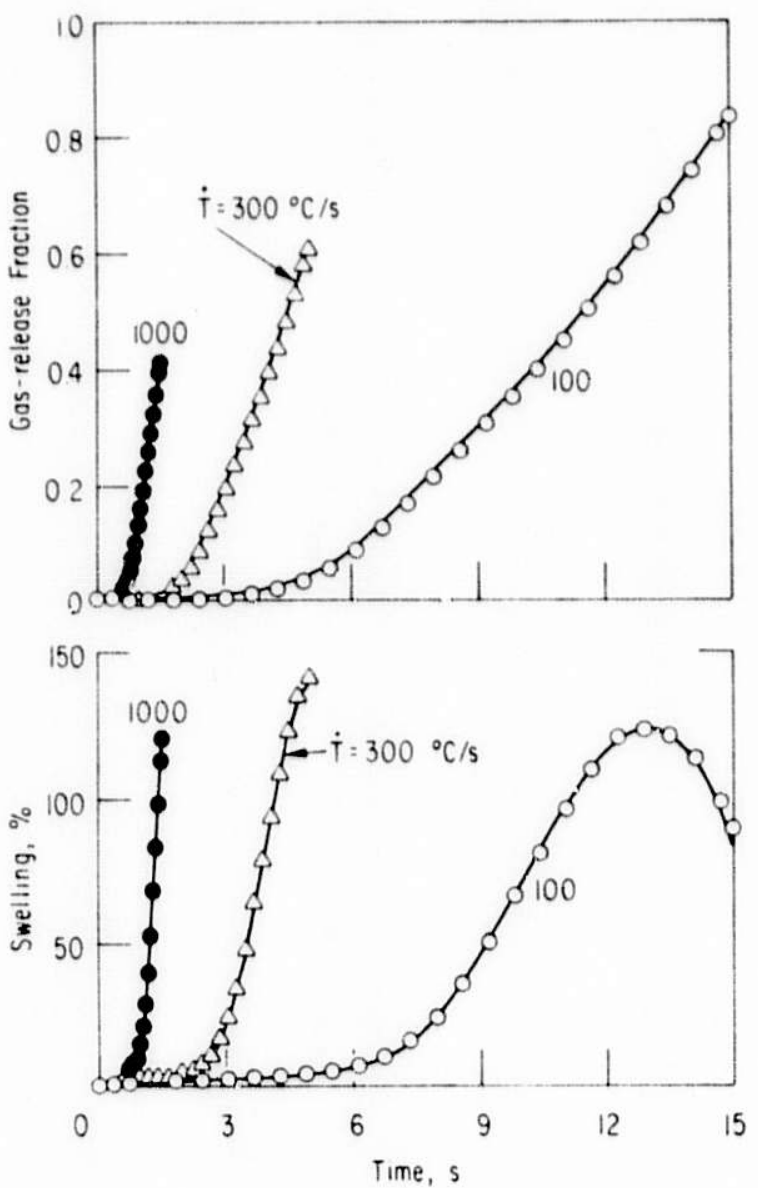

Fig. 14.

Effect of Transient Thermal Gradient on Gas Release and swelling.
Fig. 13.

Effect of Transient Heating Rate on Gas Release and Syelling.
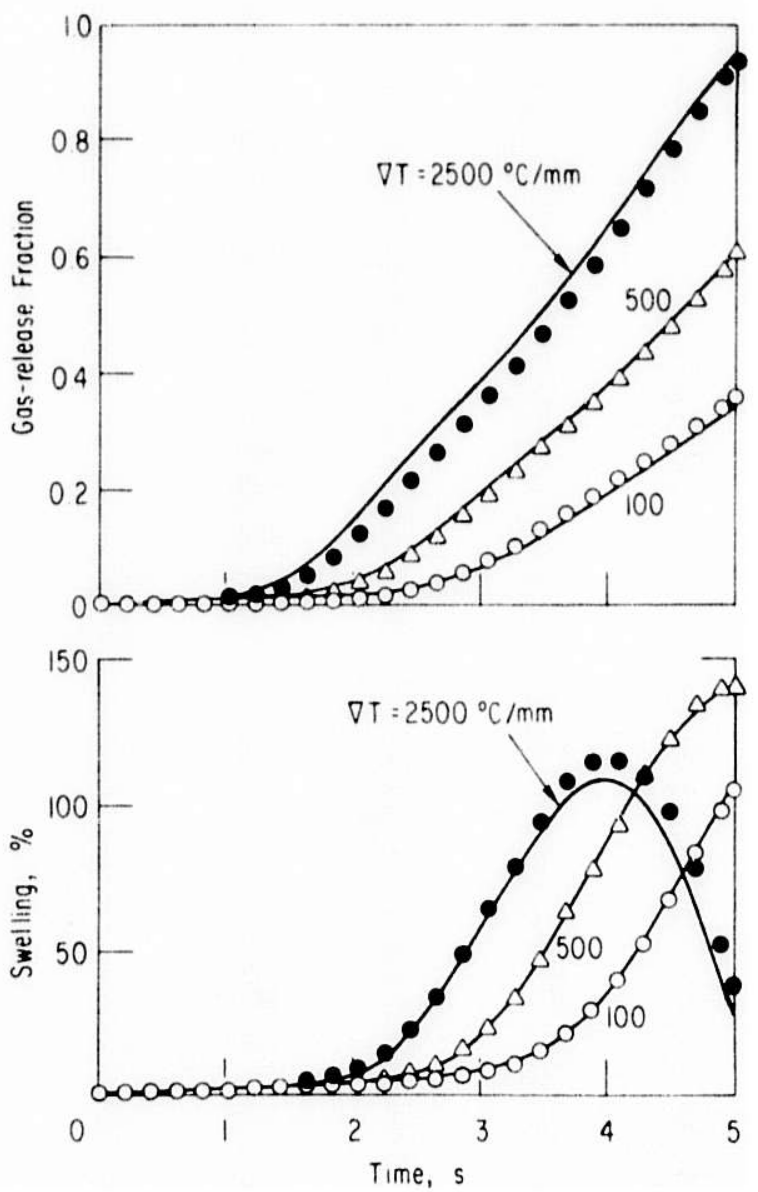

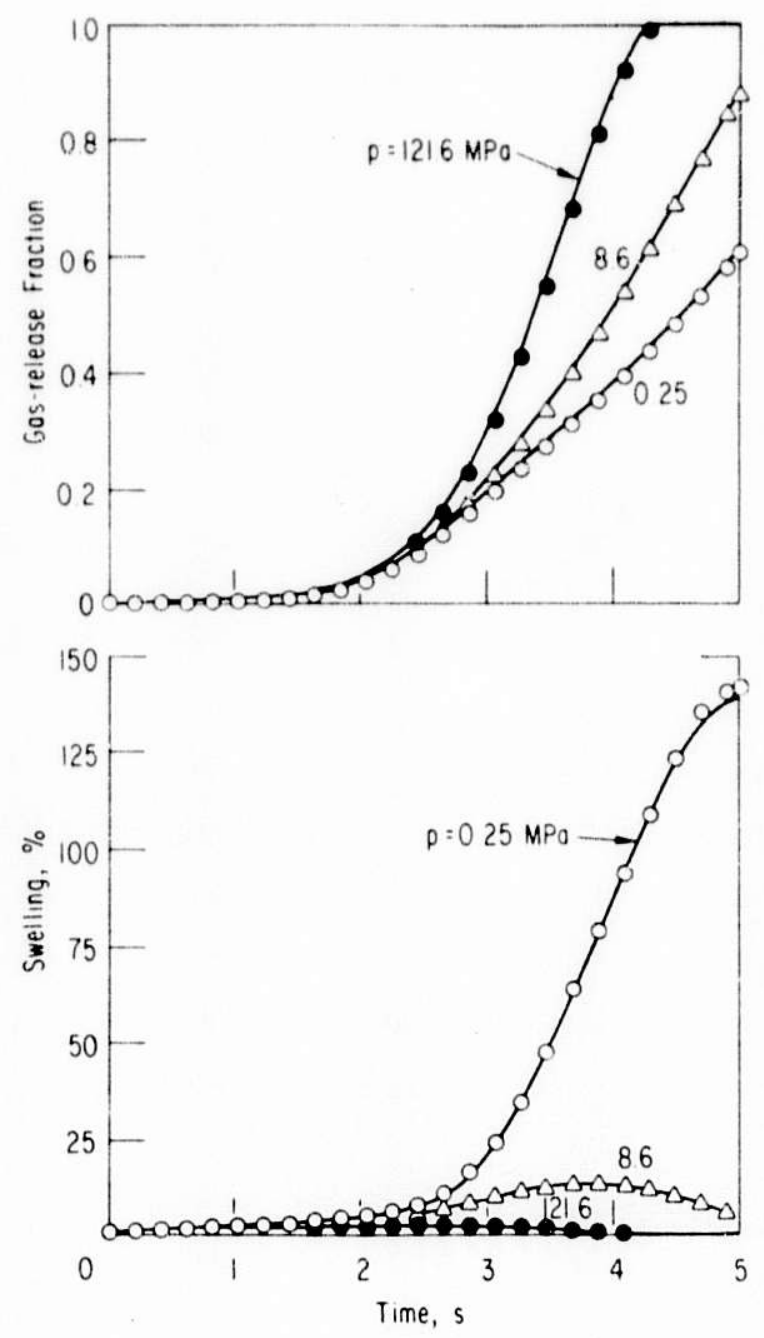

Fig. 15. Effect of Pressure on Gas Release and Swelling.

$2500^{\circ} \mathrm{C} / \mathrm{mm}$, shown in Fig. 14. Even here, the agreement is fairly good. The discrepancy is due primarily to the effects of random migration early in the transient. Random migration is included in the generalized parametric model in an implicit way by defining $\mu$ and $\phi$ in terms of total gas release and swelling. The model could be further refined to include random-migration contributions explicitly, but it is unlikely that the improvement in precision would justify the increase in complexity.

Finally, several arbitrary variations in pressure were considered to occur during a base-case ramp. Results for one of these pressure histories are shown in Fig. 16. The pressure was increased linearly from 0.25 to $121.6 \mathrm{MPa}$, from 1 to $4 \mathrm{~s}$ transient time, simulating gap closure and cladding pressurization of the fuel. From 4 to $5 \mathrm{~s}$, the pressure was reduced again to $0.25 \mathrm{MPa}$, simulating 


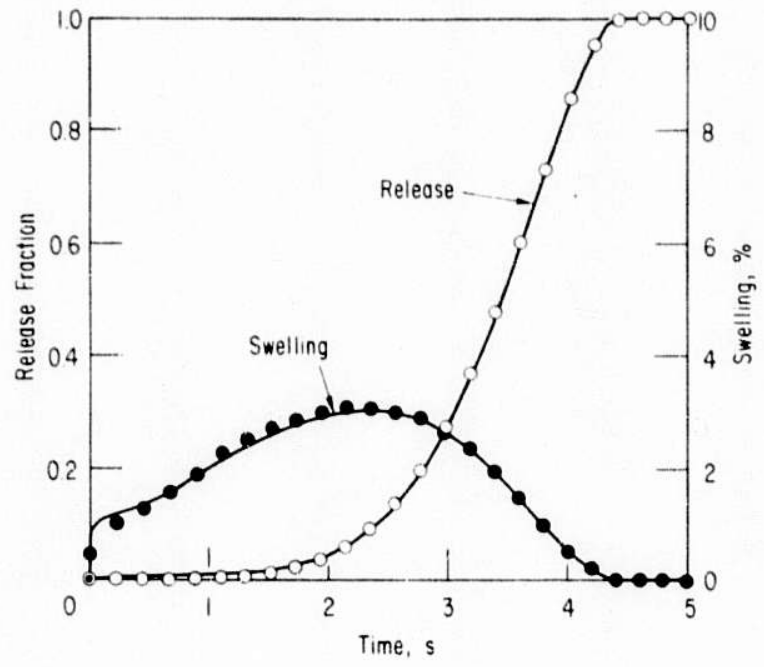

Fi.g. 16.

Effect of Hypothetical Variation in Pressure on Gas Release and Swelling in Fuel Subjected to a Thermal Transient.

gap closure and cladding pressurization of the fuel. From 4 to $5 \mathrm{~s}$, the pressure was reduced again to $0.25 \mathrm{MPa}$, simulating cladding failure and stress relaxation in the fuel. These conditions lead to very small swelling, since the pressure reduces the swelling throughout most of the transient. In addition, gas release is enhanced by the pressure and is almost complete by the time the pressure starts to fall. Little gas remains in the grains to contribute to swelling. 
V. CONCLUSTONS

A generalized parametric model has been developed to predict transient fission-gas release from, and swelling within, grains of oxide fuel. The model is developed from the basic physics of the process by which bubbles migrate in a thermal gradient by the motion of matrix atoms over the inner bubble surfaces. Although a number of simplifying assumptions are used to derive the formalism of the model, the assumptions are removed by generalizing two parameters, allowing them to vary with a dimensionless time variable and with pressure.

The variation of the parameters is determined quantitatively from the FRAS code, which explicitly calculates the same results by a detailed finitedifference analysis of the transient evolution of the bubble-size distribution. The generalized parametric model, in the form of a computer code called PFRAS, has the capability of reproducing FRAS results with good accuracy for a broad range of initial and transient conditions. In addition, the demands of the PFRAS code for computer storage and execution time are reduced by an order of magnitude compared to the requirements of the FRAS code. The PFRAS code is therefore suitable for inclusion in nulti-node pin-mechanics codes. It provides a realistic means for considering the interaction between fuel. swelling and local hydrostatic stress during a thermal transient.

The PFRAS code is developed on the assumption that the dimensionless time for biased migration is sufficient to characterize transient gas release and swelling. The effects of random migration are included implicitly, since the FRAS code considers both types of migration. However, if future experimental results indicate that the present calibration of the FRAS code overly discounts random migration, it may be necessary to include random migration explicitly in the PFRAS formalisin. It should not be difficult to incorporate this feature if it proves necessary. 


\section{APPENDIX}

\section{Calculation of Mobility and Swelling Parameters $\mu$ and $\phi$ in PFRAS}

The fundamental concept of the generalized parametric approach is that bubble mobility and swelling can be adequately characterized by two variables: the dimensionless time $\tau$, which characterizes transient history effects, and the 1ccal hydrostatic pressure $P$. These two variables uniquely determine the parameters $\mu$ and $\phi$, which, in conjunction with the variables that define the transient and fuel conditions, can be used to determine the fission-gas release and swelling.

Application of this concept in the PFRAS code requires the development of a technique to specify the dependence of each of the parameters $\mu$ and $\phi$ on the variables $\tau$ and P. Briefly, the technique used here is to generate from FRAS results a table of values of $\mu$ for specific values of $\tau$ and $P$. A two-dimensional linear interpolation is used to determine from the table values of $\mu$ for arbitrary values of $\tau$ and $P$. The same approach is used to determine $\phi$.

Values of $\mu$ for selected values of $\tau$ and $P$ are given in Table A1. These results were obtained from the FRAS results corresponding to the curves shown in Fig. 2. Each of these curves represents 50 data points. To simplify the table, or:1y 11 points were used from each curve, and these values were obtained by 1.near interpolation of $\log \mu$ as a function of $\log \tau$. The same method was used to derive the values of $\phi$, given in Table A2.

Tables A1 and A2 were used in conjunction with a two-dimensional linear interpolation to provide values of $\mu$ and $\phi$ for each time step in the PFRAS code. The interpolation procedure will be described here for the parameter $\mu$; the procedure for $\phi$ is the same.

It is assumed that linear interpolation on a logarithmic scale is appropriate; that is, that the curves shown in Fig. 2 can be approximated by straightline segments, and that similar curves obtained by plotting $\log \mu$ as a function of $\log \mathrm{P}$ for specific values of $\tau$ could be approximated in the same way. To simplify the notation, consider the change of variables

$$
\begin{aligned}
& x=\log \tau, \\
& y=\log P,
\end{aligned}
$$


Table A1. $\mu \times 10^{-5}$ for Various $\tau$ and $P$

\begin{tabular}{|c|c|c|c|c|c|c|c|c|}
\hline \multicolumn{9}{|c|}{$\mathrm{P}(\mathrm{MPa})$} \\
\hline$\tau$ & 0.1 & 0.25 & 1.0 & 3.0 & 8.6 & 20.3 & 60.8 & 142 \\
\hline 1 & 1.171 & 1.171 & 1.171 & 1.171 & 1.172 & 1.172 & 1.173 & 1.171 \\
\hline 3 & 3.429 & 3.429 & 3.431 & 3.434 & 3.444 & 3.461 & 3.505 & 3.555 \\
\hline 10 & 9.943 & 9.945 & 9.945 & 9.978 & 10.04 & 10.15 & 10.44 & 10.77 \\
\hline 30 & 24.58 & 24.59 & 24.64 & 24.75 & 25.03 & 25.53 & 26.70 & 27.98 \\
\hline 100 & 60.60 & 60.66 & 60.91 & 61.56 & 63.11 & 65.66 & 71.05 & 76.37 \\
\hline 300 & 113.8 & 114.1 & 115.6 & 119.4 & 127.5 & 139.4 & 161.7 & 181.5 \\
\hline 1000 & 140.9 & 142.8 & 151.4 & 169.8 & 205.5 & 254.1 & 342.8 & 420.9 \\
\hline 3000 & 135.3 & 141.4 & 165.2 & 209.0 & 287.0 & 390.6 & 591.3 & 01. 0 \\
\hline 10000 & 138.1 & 155.1 & 212.1 & 304.8 & 458.7 & 658.4 & 1048 & 1456 \\
\hline 30000 & 157.1 & 194.0 & 305.9 & 477.2 & 754.5 & 1110 & 1805 & 2542 \\
\hline 100000 & 203.5 & 282.4 & 500.4 & 819.6 & 1333 & 1991 & 3279 & 4648 \\
\hline
\end{tabular}


Table A2. $\phi \times 10^{30}$ for Various $\tau$ and $P$

\begin{tabular}{|c|c|c|c|c|c|c|c|c|}
\hline \multicolumn{9}{|c|}{$\mathrm{P}(\mathrm{MPa})$} \\
\hline$\tau$ & 0.1 & 0.25 & 1.0 & 3.0 & 8.6 & 20.3 & 60.8 & 142 \\
\hline 1 & 2411 & 2410 & 2405 & 2392 & 2359 & 2295 & 2123 & 1905 \\
\hline 3 & 925 & 924.4 & 921.3 & 913.4 & 893.0 & 855.5 & 761.8 & 655.3 \\
\hline 10 & 329.7 & 329.3 & 327.6 & 323.1 & 311.8 & 292.0 & 247.0 & 202.1 \\
\hline 30 & 132.4 & 132.2 & 131.1 & 128.8 & 121.5 & 110.5 & 88.28 & 68.69 \\
\hline 100 & 53.63 & 53.45 & 52.56 & 50.42 & 45.82 & 39.40 & 28.77 & 20.99 \\
\hline 300 & 30.31 & 30.02 & 28.67 & 25.88 & 21.22 & 16.39 & 10.51 & 7.122 \\
\hline 1000 & 27.30 & 26.15 & 22.07 & 16.50 & 10.71 & 6.823 & 3.580 & 2.193 \\
\hline 3000 & 27.86 & 24.53 & 16.61 & 9.970 & 5.300 & 2.932 & 1.328 & 0.7479 \\
\hline 10000 & 24.00 & 18.00 & 9.177 & 4.493 & 2.056 & 1.032 & 0.4244 & 0.2251 \\
\hline 30000 & 16.98 & 10.78 & 4.376 & 1.868 & 0.7768 & 0.3693 & 0.1437 & 0.07359 \\
\hline 100000 & 9.658 & 5.082 & 1.703 & 0.6611 & 0.2581 & 0.1179 & 0.04423 & 0.02222 \\
\hline
\end{tabular}


and

$$
z=\log \mu \text {. }
$$

The values of $\mathrm{z}$ corresponding to a rectangular grid of values of $\mathrm{x}$ and $\mathrm{y}$ can be obtained from Table Al. An element of this grid is shown schematically in Fig. Al by the points a, b, c, and d. Consider a point u on this grid,

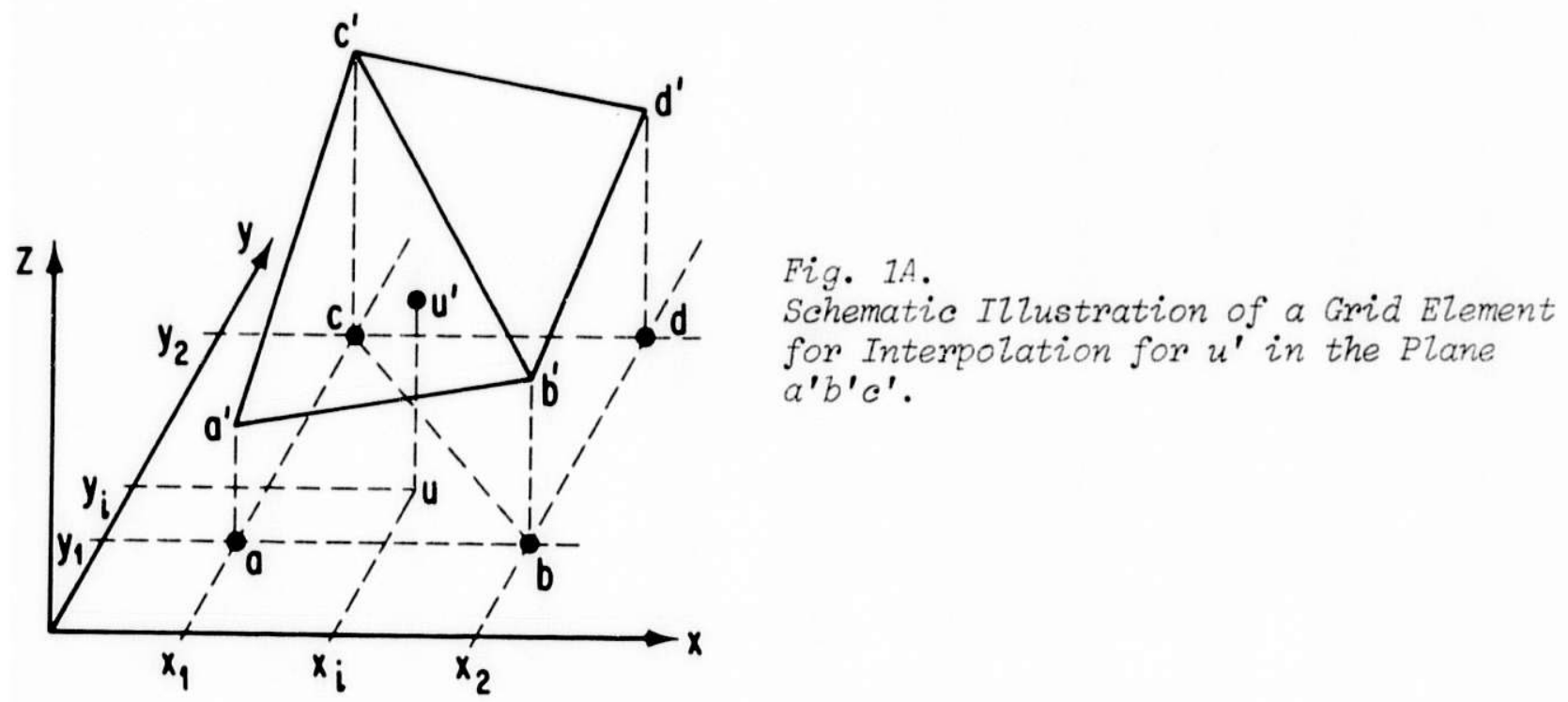

with coordinates $x_{i}, y_{i}$, as shown in the figure. This point characterizes the values of $\tau$ and $P$ for a particular time step in the transient. The corresponding value of $\mathrm{z}$ is determined by interpolation in the plane defined by the three points $\mathrm{a}^{\prime}, \mathrm{b}^{\prime}$, and $\mathrm{c}^{\prime}$. With a rectangular grid, the interpolated value can be written in the simple form

$$
z(u)=z_{0}+\Delta z x_{x} \delta+\Delta z_{y} \delta y,
$$

where $z_{0}=z(a), \Delta z_{x}=z(b)-z(a), \Delta z_{y}=z(c)-z(a)$, and $\delta x$ and $\delta y$ are the fractional changes in $x$ and $y$ relative to the grid spacing,

$$
\delta x=\frac{x_{i}-x_{1}}{x_{2}-x_{1}}
$$

and

$$
\delta y=\frac{y_{i}-y_{1}}{y_{2}-y_{1}}
$$


From Eqs. (A3) and (A4), it follows that

$$
\log \mu=\log \mu_{a}+\delta x \log \frac{\mu_{b}}{\mu_{a}}+\delta y \log \frac{\mu_{c}}{\mu_{a}},
$$

or

$$
\mu=\mu_{a}^{1-\delta x-\delta y} \mu_{b}^{\delta x} \mu_{c}^{\delta y} .
$$

If the point $\left(x_{i}, y_{i}\right)$ lies in the alternate triangle bcd, it can be shown in a similar manner that the interpolated result is given by

$$
\mu=\mu_{d} \delta x+\delta y-1 \mu_{c}^{1-\delta x} \mu_{b}^{1-\delta y}
$$

The choice of Eq. (A5) or Eq. (A6) follows very simply from the magnitude of $\delta x+\delta y$. If $\delta x+\delta y \leq 1$, triangle abc is used, and if $\delta x+\delta y>1$, triangle bcd is used.

The calculation for $\phi$ is completely analogous; one need only substitute $\phi$ for $\mu$ in Eqs. (A5) and (A6). The interpolation for both variables comprises only a small part of the PFRAS code, and its efficiency appears to be satisfactory. 


\section{ACKNOWLEDGMENTS}

A number of helpful discussions with my colleagues, particularly L. W.

Deitrich, R. H. Sevy, J. M. Kramer, R. W. Ostensen, and R. C. Wunderlich, are gratefully acknowledged. 


\section{REFERENCES}

1. E. E. Gruber, Calculation of Transient Fission-gas Release from Oxide FueZs, ANL-8143, Nov 1974.

2. E. E. Gruber, Trans. Am. Nuc1. Soc., 18, 126 (1974).

3. E. E. Gruber, Am. Cer. Soc. Bulletin, 53, 370 (1974).

4. V. Z. Jankus and R. W. Weeks, Nuc1. Eng. and Design, 18(1), 83-96 (1972).

5. E. E. Gruber, J. App1. Phys., 38, 243 (1967).

6. Am. Inst. of Physics Handbook, Ed., D. E. Gray, McGraw Hill Book Co., Inc., New York, 1963.

7. P. S. Maiya, J. Nuc1. Mat., 40, 57 (1971).

8. F. B. Hildebrand, Introduction to Numerical Analysis, McGraw Hill Book Co., Inc., New York, 1956.

9. W. M. Robertson, J. Nuc1. Mat., 30, (1967).

10. E. E. Gruber, Proc. of Int1. Conf. on Physical Metallurgy of Reactor Fuel Elements, Berkeley, U.K., Sept 1973.

11. M. E. Gu1den, J. Nuc1. Mat., 23, 30 (1967).

12. K. G. Denbigh, The Thermodynamics of the Steady State, John Wiley \& Sons, Inc., New York, 1951.

13. F. A. Nichols, J. Nuc1. Mat., 30, 143 (1969).

14. J. Crank, The Mathematics of Diffusion, Oxford Univ. Press, 1956, p. 86.

15. A. H. Booth, AECL-496, Sept 1957.

16. Standard Mathematical Tables, 18th Edition, Ed., S. M. Seiby, the Chemical Rubber Co., Cleveland, Ohio, 1960, p. 17.

17. E. E. Gruber, Trans. Am. Nuc1. Soc., 22, 418 (1975).

18. R. C. Wunderlich and E. E. Gruber, Trans. Am. Nucl. Soc., 23, 276 (1976). 